\title{
A new method for Stokes problems with circular boundaries using degenerate kernel and Fourier series
}

\author{
Jeng-Tzong Chen ${ }^{1, * \dagger}$, Chia-Chun $\mathrm{Hsiao}^{1}$ and Shyue-Yuh Leu ${ }^{2}$ \\ ${ }^{1}$ Department of Harbor and River Engineering, National Taiwan Ocean University, Keelung, Taiwan \\ ${ }^{2}$ Department of Aviation Mechanical Engineering, China Institute of Technology, Taipei, Taiwan
}

\begin{abstract}
SUMMARY
This study is concerned with the Stokes flow of an incompressible fluid of constant density and viscosity with circular boundaries. To fully capture the circular boundary, the boundary densities in the direct and indirect boundary integral equations (BIEs) are expanded in terms of Fourier series. The kernel functions in either the direct BIE or the indirect BIE are expanded to degenerate kernels by using the separation of field and source points. Consequently, the improper integrals are transformed to series sum and are easily calculated. The linear algebraic system can be established by matching the boundary conditions at the collocation points. Then, the unknown Fourier coefficients can be easily determined. Finally, several examples including circular and eccentric domains are presented to demonstrate the validity of the present method. Five gains were obtained: (1) meshless approach; (2) free of boundary-layer effect; (3) singularity free; (4) exponential convergence; and (5) well-posed model. Copyright (C) 2007 John Wiley \& Sons, Ltd.
\end{abstract}

Received 5 May 2007; Revised 26 September 2007; Accepted 3 October 2007

KEY WORDS: biharmonic equation; boundary integral equation; null-field integral equation; degenerate kernel; Fourier series; Stokes flow

\section{INTRODUCTION}

The boundary element method (BEM) by discretizing the boundary integral equation (BIE) has been extensively applied for engineering problems recently more than domain-type methods, e.g. finite element method (FEM) or finite difference method. It is noted that improper integrals on the boundary should be handled particularly when BEM is used. In the past, many researchers proposed several regularization techniques to deal with the singularity and hypersingularity. To determine the Cauchy principal value and the Hadamard principal value in the singular and hypersingular integrals is a critical issue in BEM/BIE method (BIEM). The technique of the integration by parts

\footnotetext{
*Correspondence to: Jeng-Tzong Chen, Department of Harbor and River Engineering, National Taiwan Ocean University, Keelung, Taiwan.

†E-mail: jtchen@mail.ntou.edu.tw
} 
to reduce the order of singularity [1] is an alternative. One order of singularity is shifted to the density function from the kernel. In this paper, instead of using the previous concepts, the kernel function is described in an analytical form on each side across the boundary (interior and exterior) by employing the degenerate kernel since the potential is discontinuous across the boundary. Therefore, degenerate kernel, namely separable kernel, is a vital tool to study the Stokes problems with circular boundaries. Boundary integral formulation is nothing more than the linear algebra once fundamental solutions are expressed by separable kernels. One gain is that this formulation is free of singularity.

BIEs for the plate problems were acquired from the Rayleigh-Green identity $[2,3]$ and the null-field integral equations were derived by collocating the field point outside the domain. The formulation for the plate problems can be applied to study the Stokes flow problem since both displacement and stream function satisfy biharmonic equation. The kernel functions in the present formulation are expanded to degenerate kernels in an analytical series representation by separating the source point and field point and the boundary densities are expressed in terms of Fourier series. It is well known that Fourier series is always incorporated to formulate the solution for problems with circular boundaries [4,5]. Bird and Steele [4] presented a Fourier series procedure to solve circular plate problems containing multiple circular holes. Also, Mogilevskaya and Crouch $[6,7]$ presented a method in conjunction with Fourier series for solving problems with randomly distributed circular elastic inclusions with arbitrary properties. Although Fourier series expansions have been employed, it seems that no one has ever introduced the degenerate kernel in BIEs to tackle the problem. Therefore, the BIE in conjunction with degenerate kernel and Fourier series is proposed to solve the Stokes problems with circular boundaries. Two gains are that exponential convergence instead of linear algebraic order can be obtained and mesh generation on the boundary is not required.

The Stokes flow problem with circular boundaries is considered since the stream function as well as displacement of plate problem satisfies the biharmonic equation. The computation for internal Stokes flow for a circle by integral equations was solved analytically [8]. Later, Chen et al. revisited this problem and obtained the series solution by using degenerate kernel and Fourier series [2]. A spectral boundary element approach to three-dimensional Stokes flow was proposed by Muldowney and Higdon [9]. A numerical approach for Stokes flow past a particle of arbitrary shape was proposed by Youngren and Acrivos [10]. The flow between eccentric cylinders for the doubly-connected problem is focused in this paper. Many papers were published on these problems, some important works are those of Kamal [11], DiPrima and Stuart [12]. Ingham and Kelmanson [13], Kelmanson [14] and Wannier [15] also applied the BIE to solve the problems of two-dimensional steady slow flow for the lubrication technology. Although both of the Kelmanson's formulation and the present method are based on the same BIE, the main differences are pointed out here. First, the kernel functions in Kelmanson's paper are fundamental solutions instead of degenerate kernels. It is noted that all the improper integrals are transformed to series sum and are easily calculated when the degenerate kernels are used since the potential across the boundary can be described explicitly in both sides, interior and exterior regions. Second, Fourier expansion for the boundary density is used in this paper instead of linear boundary element scheme $[13,14]$.

The purpose of this paper is to study biharmonic problems with circular boundaries by using direct and indirect BIEs in conjunction with degenerate kernels, Fourier series, vector decomposition and the adaptive observer frame. It is very convenient to be able to expand the solution in an alternative form, each form referring to a different specified coordinate set describing the 
same solution. In the polar coordinate system, the calculation of potential gradients in the normal and tangential directions for the non-concentric domain must be taken care of. Therefore, the technique of vector decomposition is adopted to deal with the problem for the non-concentric case. It is interesting that Stokes flow problem (not involving the Poisson ratio) can also be solved by using the present formulation for plate although the Poisson ratio is contained in the approach. Although the well-known alternative BIE formulations for these problems [16] have been explored, the indirect BIE as well as the direct BIE in conjunction with degenerate kernels and Fourier series are both used to solve the Stokes problems. Single- and double-layer potentials are simultaneously used to construct the indirect BIE. Although the indirect method cannot provide the null-field integral equation, the compatible relationship of the boundary data (single- and double-layer fictitious densities) is obtained by moving the domain point in BIE to the boundary. Special care must be taken in selecting the appropriate expressions (interior and exterior) for the kernel function. Regarding the direct BIE, we employ the concept of null-field integral equations and collocate the point on the real boundary in real implementation. Finally, several examples are presented to show the validity of the present method and some conclusions are made.

\section{FORMULATION OF THE STOKES FLOW PROBLEMS}

The governing equation of Stokes flow is derived from the Navier-Stokes equation as follows:

$$
\rho \frac{\mathrm{D} \underset{\sim}{\mathrm{D} t}}{\underset{\mathrm{D}}{V}}=\rho g-\nabla P+\mu \nabla^{2} \underset{\sim}{V}
$$

where $\underset{\sim}{V}$ denotes the velocity field $\underset{\sim}{V}=\left(v_{r}, v_{\theta}\right), \rho$ the density of fluid, $t$ the time, $g$ the gravity, $P$ the pressure and $\mu$ the viscosity. Therefore, the first term of Equation (1) means inertia force, the second term denotes body force, the third term is pressure gradient and the final term is viscous force. The term of inertia force can be neglected since the low Reynolds number flow is considered (inertia force « viscous force) and the body force is also neglected to reduce Equation (1) as follows:

$$
\nabla P=\mu \nabla^{2} \underset{\sim}{V}
$$

The continuity equation for the incompressible two-dimensional flow is expressed as follows:

$$
\frac{1}{r} \frac{\partial\left(r v_{r}\right)}{\partial r}+\frac{1}{r} \frac{\partial v_{\theta}}{\partial \theta}=0
$$

and the velocity components, $v_{r}$ and $v_{\theta}$, can be related to the stream function $u(r, \theta)$ through the equations

$$
\begin{aligned}
& v_{r}=\frac{1}{r} \frac{\partial u}{\partial \theta} \\
& v_{\theta}=-\frac{\partial u}{\partial r}
\end{aligned}
$$




\section{J.-T. CHEN, C.-C. HSIAO AND S.-Y. LEU}

The biharmonic equation can be derived by associating Equations (2)-(5) as follows:

$$
\nabla^{4} u=0
$$

Introducing the vorticity as the Laplacian of the stream function $u[13,14]$, we have

$$
\begin{aligned}
\nabla^{2} u & =\omega \\
\nabla^{2} \omega & =0
\end{aligned}
$$

where $\omega$ is the vorticity. To deal with the Stokes problem, two ways are used in the literature $[3,14]$. First, the biharmonic equation of Equation (6) is treated [3]. The other one is solving the Poisson and Laplace equation in Equations (7)-(8) [14]. In this paper, we focus on the former formulation.

\section{DIRECT BIE METHOD}

\subsection{BIE for the domain point}

Here, we use plate formulation to solve Stokes problems since they both satisfy the biharmonic equation. The direct BIEs for the domain point can be derived from the Rayleigh-Green identity $[2,3]$ as follows:

$$
\begin{aligned}
8 \pi u(x)= & -\int_{B} U(s, x) v(s) \mathrm{d} B(s)+\int_{B} \Theta(s, x) m(s) \mathrm{d} B(s) \\
& -\int_{B} M(s, x) \theta(s) \mathrm{d} B(s)+\int_{B} V(s, x) u(s) \mathrm{d} B(s), \quad x \in \Omega \\
8 \pi \theta(x)=- & \int_{B} U_{\theta}(s, x) v(s) \mathrm{d} B(s)+\int_{B} \Theta_{\theta}(s, x) m(s) \mathrm{d} B(s) \\
& -\int_{B} M_{\theta}(s, x) \theta(s) \mathrm{d} B(s)+\int_{B} V_{\theta}(s, x) u(s) \mathrm{d} B(s), \quad x \in \Omega \\
8 \pi m(x)= & -\int_{B} U_{m}(s, x) v(s) \mathrm{d} B(s)+\int_{B} \Theta_{m}(s, x) m(s) \mathrm{d} B(s) \\
& -\int_{B} M_{m}(s, x) \theta(s) \mathrm{d} B(s)+\int_{B} V_{m}(s, x) u(s) \mathrm{d} B(s), \quad x \in \Omega \\
& -\int_{B} U_{v}(s, x) v(s) \mathrm{d} B(s)+\int_{B} \Theta_{v}(s, x) m(s) \mathrm{d} B(s) \\
& \\
8 \pi v(x)= &
\end{aligned}
$$




\section{A NEW METHOD FOR STOKES PROBLEMS WITH CIRCULAR BOUNDARIES}

where $B$ is the boundary of the domain $\Omega, u(x), \theta(x), m(x)$ and $v(x)$ are the displacement, slope, moment and shear force for solid mechanics, $s$ and $x$ are the source point and field point, respectively. However, $u(x)$ is defined as the stream function in this paper instead of displacement for plate problem. The kernel functions $U, \Theta, M, V, U_{\theta}, \Theta_{\theta}, M_{\theta}, V_{\theta}, U_{m}, \Theta_{m}, M_{m}, V_{m}, U_{v}, \Theta_{v}$, $M_{v}, V_{v}$ in Equations (9)-(12) are expanded to degenerate kernels by using the separation of source and field points $[3,17]$. The kernel function $U(s, x)$ in Equation (9) is the fundamental solution that satisfies

$$
\nabla^{4} U(s, x)=8 \pi \delta(s-x)
$$

where $\delta(s-x)$ is the Dirac-delta function. Therefore, the fundamental solution can be obtained

$$
U(s, x)=r^{2} \ln r
$$

where $r$ is the distance between source point $s$ and field point $x$. The relationship among $u(x), \theta(x), m(x)$ and $v(x)$ are shown as follows:

$$
\begin{aligned}
& \theta(x)=K_{\theta, x}(u(x))=\frac{\partial u(x)}{\partial n_{x}} \\
& m(x)=K_{m, x}(u(x))=v \nabla_{x}^{2} u(x)+(1-v) \frac{\partial^{2} u(x)}{\partial^{2} n_{x}} \\
& v(x)=K_{v, x}(u(x))=\frac{\partial \nabla_{x}^{2} u(x)}{\partial n_{x}}+(1-v) \frac{\partial}{\partial t_{x}}\left[\frac{\partial}{\partial n_{x}}\left(\frac{\partial u(x)}{\partial t_{x}}\right)\right]
\end{aligned}
$$

where $K_{\theta, x}(\cdot), K_{m, x}(\cdot), K_{v, x}(\cdot)$ are the slope, moment and shear force operators with respect to the point $x, \partial / \partial n_{x}$ is the normal derivative with respect to the field point $x, \partial / \partial t_{x}$ is the tangential derivative with respect to the field point $x, \nabla_{x}^{2}$ means the Laplacian operator and $v$ is the Poisson's ratio.

By taking the Laplacian with respect to $u(x)$ in Equation (9), the vorticity function is derived as follows:

$$
\begin{aligned}
8 \pi \omega(x)= & -\int_{B} U_{\nabla^{2}}(s, x) v(s) \mathrm{d} B(s)+\int_{B} \Theta_{\nabla^{2}}(s, x) v(s) \mathrm{d} B(s) \\
& -\int_{B} M_{\nabla^{2}}(s, x) v(s) \mathrm{d} B(s)+\int_{B} V_{\nabla^{2}}(s, x) v(s) \mathrm{d} B(s), \quad x \in \Omega
\end{aligned}
$$

where $U_{\nabla^{2}}(s, x), \Theta_{\nabla^{2}}(s, x), M_{\nabla^{2}}(s, x)$ and $V_{\nabla^{2}}(s, x)$ are the Laplacian of degenerate kernels $U(s, x), \Theta(s, x), M(s, x)$ and $V(s, x)$, respectively. The kernel functions are listed in Appendix A. By using the formulations in conjunction with the degenerate kernels, Fourier series and adaptive observer system, the stream function and vorticity can be solved. 


\subsection{Null-field integral equation}

The null-field integral equations were obtained by collocating the field point $x$ outside the domain as follows:

$$
\begin{aligned}
0= & -\int_{B} U(s, x) v(s) \mathrm{d} B(s)+\int_{B} \Theta(s, x) m(s) \mathrm{d} B(s) \\
& -\int_{B} M(s, x) \theta(s) \mathrm{d} B(s)+\int_{B} V(s, x) u(s) \mathrm{d} B(s), \quad x \in \Omega^{\mathrm{C}} \\
0= & -\int_{B} U_{\theta}(s, x) v(s) \mathrm{d} B(s)+\int_{B} \Theta_{\theta}(s, x) m(s) \mathrm{d} B(s) \\
& -\int_{B} M_{\theta}(s, x) \theta(s) \mathrm{d} B(s)+\int_{B} V_{\theta}(s, x) u(s) \mathrm{d} B(s), \quad x \in \Omega^{\mathrm{C}} \\
0= & \int_{B} U_{m}(s, x) v(s) \mathrm{d} B(s)+\int_{B} \Theta_{m}(s, x) m(s) \mathrm{d} B(s) \\
& -\int_{B} M_{m}(s, x) \theta(s) \mathrm{d} B(s)+\int_{B} V_{m}(s, x) u(s) \mathrm{d} B(s), \quad x \in \Omega^{\mathrm{C}} \\
0= & \int_{B} U_{v}(s, x) v(s) \mathrm{d} B(s)+\int_{B} \Theta_{v}(s, x) m(s) \mathrm{d} B(s) \\
& -\int_{B} M_{v}(s, x) \theta(s) \mathrm{d} B(s)+\int_{B} V_{v}(s, x) u(s) \mathrm{d} B(s), \quad x \in \Omega^{\mathrm{C}}
\end{aligned}
$$

where $\Omega^{\mathrm{C}}$ is the complementary domain of $\Omega$. Since the four equations of Equations (19)-(22) are given, there are six $\left(C_{2}^{4}\right)$ options for choosing any two equations to solve the problems. For simplicity, Equations (19) and (20) are used. In the real implementation, the collocation point in the null-field integral equation is moved to the boundary from $\Omega^{\mathrm{C}}$ such that the kernel functions can be expressed in terms of appropriate forms of degenerate kernels. Consequently, all the improper integrals disappear and are transformed to series sum in the BIEs since the potential across the boundary can be described explicitly in both sides by using degenerate kernels.

\subsection{Expansion of Fourier series}

The boundary densities $u(s), \theta(s), m(s)$ and $v(s)$ are expressed in terms of Fourier series as follows:

$$
\begin{aligned}
& u(s)=p_{0}+\sum_{n=1}^{M}\left(p_{n} \cos n \theta+q_{n} \sin n \theta\right) \\
& \theta(s)=g_{0}+\sum_{n=1}^{M}\left(g_{n} \cos n \theta+h_{n} \sin n \theta\right)
\end{aligned}
$$




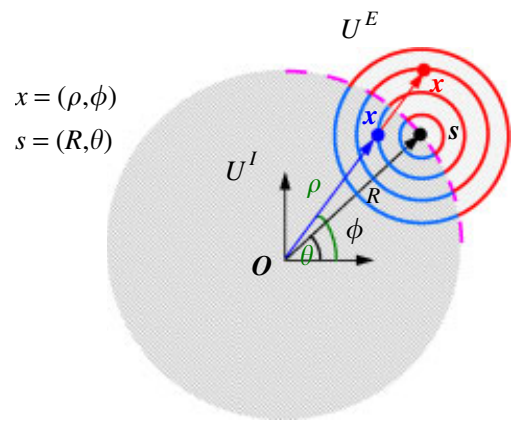

Figure 1. Degenerate kernel for $U(s, x)$.

$$
\begin{gathered}
m(s)=c_{0}+\sum_{n=1}^{M}\left(c_{n} \cos n \theta+d_{n} \sin n \theta\right) \\
v(s)=a_{0}+\sum_{n=1}^{M}\left(a_{n} \cos n \theta+b_{n} \sin n \theta\right)
\end{gathered}
$$

where $a_{0}, a_{n}, b_{n}, c_{0}, c_{n}, d_{n}, g_{0}, g_{n}, h_{n}, p_{0}, p_{n}$ and $q_{n}$ are Fourier coefficients and $M$ denotes the number of truncated terms of Fourier series.

\subsection{Expansion of kernels}

By employing the separation technique for source and field points, the kernel function $U(s, x)$ can be expanded in terms of degenerate kernel in a series form [17] as shown below:

$$
\begin{aligned}
& U(s, x)=r^{2} \ln r \\
& =\left\{\begin{aligned}
U^{I}(s, x)= & \rho^{2}(1+\ln R)+R^{2} \ln R-\left[R \rho(1+2 \ln R)+\frac{1}{2} \frac{\rho^{3}}{R}\right] \cos (\theta-\phi) \\
& -\sum_{m=2}^{\infty}\left[\frac{1}{m(m+1)} \frac{\rho^{m+2}}{R^{m}}-\frac{1}{m(m-1)} \frac{\rho^{m}}{R^{m-2}}\right] \cos [m(\theta-\phi)], \quad R \geqslant \rho \\
U^{\mathrm{E}}(s, x)= & R^{2}(1+\ln \rho)+\rho^{2} \ln \rho-\left[\rho R(1+2 \ln \rho)+\frac{1}{2} \frac{R^{3}}{\rho}\right] \cos (\theta-\phi) \\
& -\sum_{m=2}^{\infty}\left[\frac{1}{m(m+1)} \frac{R^{m+2}}{\rho^{m}}-\frac{1}{m(m-1)} \frac{R^{m}}{\rho^{m-2}}\right] \cos [m(\theta-\phi)], \quad \rho>R
\end{aligned}\right.
\end{aligned}
$$

where the superscripts ' $I$ ' and ' $E$ ' denote the interior and exterior cases of $U(s, x)$ kernel depending on the geometry as shown in Figure 1. It is interesting to find that interior and exterior Trefftz bases are imbedded in the degenerate kernel. The other kernels in the BIEs can be obtained by utilizing the operators of Equations (15)-(17) with respect to the $U(s, x)$ kernel. The degenerate kernels $U, \Theta, M, V, U_{\theta}, \Theta_{\theta}, M_{\theta}$ and $V_{\theta}$ in Equations (9) and (10) are listed in Appendix A. It is 


\section{J.-T. CHEN, C.-C. HSIAO AND S.-Y. LEU}

noted that the interior and exterior cases of $U, \Theta, M, U_{\theta}$ and $\Theta_{\theta}$ are the same when they both approach the boundary $(\rho=R)$, since the degenerate kernels are continuous functions across the boundary. Then, the kernel function with the superscript ' $I$ ' is chosen while the field point is inside the circular region; otherwise, the kernels with the superscript ' $E$ ' are chosen.

\section{INDIRECT BIE METHOD}

Indirect BIE method is originated from the physical concept of superposition and must satisfy not only the governing equation but also the boundary conditions. There are four kinds of potentials, single-, double-, triple- and quadruple-layer potentials in the indirect BIEM for the Stokes flow problems. By choosing any two potentials, six options $\left(C_{2}^{4}\right)$ (single-double-layer potentials, singletriple-layer potentials, single-quadruple-layer potentials, double-triple-layer potentials, doublequadruple-layer potentials and triple-quadruple-layer potentials) can be chosen. For simplicity, single- and double-layer potentials are chosen here as follows:

$$
u(x)=\int_{B} U(s, x) \Phi(s) \mathrm{d} B(s)+\int_{B} \Theta(s, x) \Psi(s) \mathrm{d} B(s), \quad x \in \Omega
$$

where $\Phi(s)$ and $\Psi(s)$ are the single- and double-layer fictitious densities, respectively, and $B$ is the boundary of the domain $\Omega$. By taking normal derivative with respect to $u(x)$ in Equation (28), we have

$$
\theta(x)=\int_{B} U_{\theta}(s, x) \Phi(s) \mathrm{d} B(s)+\int_{B} \Theta_{\theta}(s, x) \Psi(s) \mathrm{d} B(s), \quad x \in \Omega
$$

The single- and double-layer fictitious densities in Equations (28) and (29) are expressed in terms of Fourier series as follows:

$$
\begin{aligned}
& \Phi(s)=a_{0}+\sum_{n=1}^{M}\left(a_{n} \cos n \theta+b_{n} \sin n \theta\right) \\
& \Psi(s)=c_{0}+\sum_{n=1}^{M}\left(c_{n} \cos n \theta+d_{n} \sin n \theta\right)
\end{aligned}
$$

where $a_{0}, a_{n}, b_{n}, c_{0}, c_{n}$ and $d_{n}$ are the Fourier coefficients and $M$ denotes the number of truncated terms of Fourier series. By taking the Laplacian with respect to $u(x)$ in Equation (28), the vorticity function is derived as shown below:

$$
\omega(x)=\int_{B} U_{\nabla^{2}}(s, x) \Phi(s) \mathrm{d} B(s)+\int_{B} \Theta_{\nabla^{2}}(s, x) \Psi(s) \mathrm{d} B(s), \quad x \in \Omega
$$

where $U_{\nabla^{2}}(s, x)$ and $\Theta_{\nabla^{2}}(s, x)$ are the Laplacian of the degenerate kernels $U(s, x)$ and $\Theta(s, x)$, respectively. It is noted that null-field integral equation in the indirect method is not available. However, the compatible relationship of boundary data can be obtained by moving the domain point $x$ in Equations (28) and (29) to the boundary $B^{-}$and $B^{+}$from inside and outside domains, respectively. 


\section{ADAPTIVE OBSERVER SYSTEM AND VECTOR DECOMPOSITION FOR THE NORMAL DERIVATIVE}

\subsection{Adaptive observer system}

Consider a biharmonic problem with circular boundaries as shown in Figure 2. Since the BIEs are frame indifferent due to objectivity, an adaptive observer system is chosen to fully employ the circular property by expanding the kernels into degenerate forms. The origin of the observer system can be adaptively located on the center of the corresponding boundary contour under integration. The dummy variable in the circular contour integration is the angle $(\theta)$ instead of radial coordinate $(R)$. By using the adaptive system, all the boundary integrals can be determined analytically free of principal value senses.

\subsection{Vector decomposition}

Since the higher-order singular equation is also one alternative to deal with the Stokes problem, potential gradient or higher-order gradient is required to calculate carefully. For the non-concentric case, special treatment for the potential gradient should be taken care as the source and field points locate on different boundaries. As shown in Figure 3, the true normal direction with respect to the collocation point $x$ on the $B_{i}$ boundary can be superimposed by using the radial direction $\underset{\sim}{e} \rho$ and angular direction $\underset{\sim}{\underset{\phi}{e}}$ on the $B_{j}$ boundary. The degenerate kernels for the higher-order singular equation ( $\theta$-formulation) are changed to

$$
\begin{aligned}
U_{n}(s, x) & =\frac{\partial U(s, x)}{\partial n_{x}} \cos \left(\phi-\phi^{\prime}\right)+\frac{\partial U(s, x)}{\partial t_{x}} \cos \left(\frac{\pi}{2}-\phi+\phi^{\prime}\right) \\
\Theta_{n}(s, x) & =\frac{\partial \Theta(s, x)}{\partial n_{x}} \cos \left(\phi-\phi^{\prime}\right)+\frac{\partial \Theta(s, x)}{\partial t_{x}} \cos \left(\frac{\pi}{2}-\phi+\phi^{\prime}\right)
\end{aligned}
$$

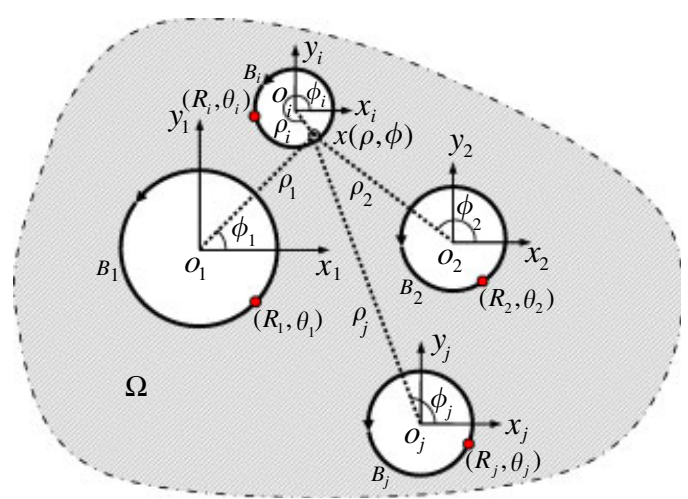

Figure 2. Adaptive observer system at $O_{j}\left(j=1,2,3, \ldots, N_{\mathrm{c}}\right)$ when integrating the corresponding circular boundary $B_{j}$ for the collocation null-field point near $B_{i}$. 


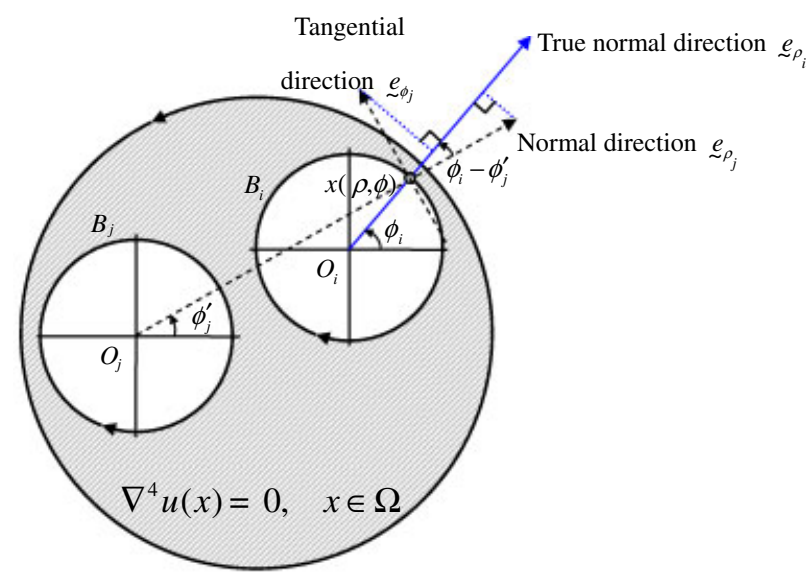

Figure 3. Vector decomposition (Collocation on $x$ and integration on $B_{j}$ ).

$$
\begin{aligned}
& M_{n}(s, x)=\frac{\partial M(s, x)}{\partial n_{x}} \cos \left(\phi-\phi^{\prime}\right)+\frac{\partial M(s, x)}{\partial t_{x}} \cos \left(\frac{\pi}{2}-\phi+\phi^{\prime}\right) \\
& V_{n}(s, x)=\frac{\partial V(s, x)}{\partial n_{x}} \cos \left(\phi-\phi^{\prime}\right)+\frac{\partial V(s, x)}{\partial t_{x}} \cos \left(\frac{\pi}{2}-\phi+\phi^{\prime}\right)
\end{aligned}
$$

The tangential derivative $\partial / \partial t_{x}$ with respect to the field point $x$ for the four kernels need to be additionally derived and are listed in Appendix A, where the normal derivative $\partial / \partial n_{x}$ is $\partial / \partial \rho$ and has been derived in the $U_{\theta}, \Theta_{\theta}, M_{\theta}$ and $V_{\theta}$ kernels. We call this treatment 'vector decomposition technique'. By approaching the collocation point from $\Omega^{\mathrm{C}}$ to $B_{i}$ and integrating the $B_{j}$ circle using the adaptive observer system of origin $O_{j}$, the normal and tangent derivatives can be superimposed as follows:

$$
\begin{aligned}
\frac{\partial}{\partial \rho_{i}} & =\cos \left(\phi_{i}-\phi_{j}^{\prime}\right) \frac{\partial}{\partial \rho_{j}}+\cos \left(\frac{\pi}{2}-\phi_{i}+\phi_{j}^{\prime}\right) \frac{1}{\rho_{j}} \frac{\partial}{\partial \phi_{j}} \\
\frac{1}{\rho_{i}} \frac{\partial}{\partial \phi_{i}} & =\cos \left(\frac{\pi}{2}-\phi_{i}+\phi_{j}^{\prime}\right) \frac{\partial}{\partial \rho_{j}}+\cos \left(\phi_{i}-\phi_{j}^{\prime}\right) \frac{1}{\rho_{j}} \frac{\partial}{\partial \phi_{j}}
\end{aligned}
$$

\section{SOLUTION PROCEDURES OF THE SEMI-ANALYTICAL APPROACHES}

Two semi-analytical approaches, the direct and indirect BIEMs are described. Direct BIEM employs the concept of the null-field integral equation but collocates on the real boundary and the indirect BIEM obtains the compatible relation of boundary data by collocating the point to the boundary from the BIE of domain point. 


\section{A NEW METHOD FOR STOKES PROBLEMS WITH CIRCULAR BOUNDARIES}

\subsection{Direct formulation}

6.1.1. Eccentric case (doubly-connected domain). By using the null-field integral equations (19)(20) as shown in Figures 4 and 5, the linear algebraic system can be constructed as follows:

$$
\left[\begin{array}{cccc}
\mathbf{U} 11 & \boldsymbol{\Theta} 11 & \mathbf{U} 12 & \boldsymbol{\Theta} 12 \\
\mathbf{U} 11_{\boldsymbol{\theta}} & \boldsymbol{\Theta} 11_{\boldsymbol{\theta}} & \mathbf{U} 12_{\boldsymbol{\theta}} & \boldsymbol{\Theta} 12_{\boldsymbol{\theta}} \\
\mathbf{U} 21 & \boldsymbol{\Theta} 21 & \mathbf{U} 22 & \boldsymbol{\Theta} 22 \\
\mathbf{U} 21_{\boldsymbol{\theta}} & \boldsymbol{\Theta} 21_{\boldsymbol{\theta}} & \mathbf{U} 22_{\boldsymbol{\theta}} & \boldsymbol{\Theta} 22_{\boldsymbol{\theta}}
\end{array}\right]\left\{\begin{array}{c}
\mathbf{v}_{1} \\
\mathbf{m}_{1} \\
\mathbf{v}_{2} \\
\mathbf{m}_{2}
\end{array}\right\}=\left[\begin{array}{cccc}
\mathbf{M} 11 & \mathbf{V} 11 & \mathbf{M} 12 & \mathbf{V} 12 \\
\mathbf{M} 11_{\boldsymbol{\theta}} & \mathbf{V} 11_{\boldsymbol{\theta}} & \mathbf{M} 12_{\boldsymbol{\theta}} & \mathbf{V} 12_{\boldsymbol{\theta}} \\
\mathbf{M} 21 & \mathbf{V} 21 & \mathbf{M} 22 & \mathbf{V} 22 \\
\mathbf{M} 21_{\boldsymbol{\theta}} & \mathbf{V} 21_{\boldsymbol{\theta}} & \mathbf{M} 22_{\boldsymbol{\theta}} & \mathbf{V} 22_{\boldsymbol{\theta}}
\end{array}\right]\left\{\begin{array}{l}
\boldsymbol{\theta}_{1} \\
\mathbf{u}_{1} \\
\boldsymbol{\theta}_{2} \\
\mathbf{u}_{2}
\end{array}\right\}
$$

For brevity, a unified form [Uij] $(i=1,2$ and $j=1,2)$ denotes the response of $U(s, x)$ kernel at the $i$ th circle point due to the source at the $j$ th circle. Otherwise, the same definition for $[\boldsymbol{\Theta} i j],[\mathbf{M} i j],[\mathbf{V} i j],\left[\mathbf{U} i j_{\theta}\right],\left[\boldsymbol{\Theta} i j_{\boldsymbol{\theta}}\right],\left[\mathbf{M} i j_{\boldsymbol{\theta}}\right]$ and $\left[\mathbf{V} i j_{\boldsymbol{\theta}}\right]$ cases. The sub-matrices $[\mathbf{U} i j],[\boldsymbol{\Theta} i j],[\mathbf{M} i j],[\mathbf{V} i j],\left[\mathbf{U} i j_{\boldsymbol{\theta}}\right],\left[\boldsymbol{\Theta} i j_{\boldsymbol{\theta}}\right],\left[\mathbf{M} i j_{\boldsymbol{\theta}}\right]$ and $\left[\mathbf{V} i j_{\boldsymbol{\theta}}\right]$ are defined as follows:

$$
\begin{aligned}
& {[\Theta i j]=\left[\begin{array}{cccccc}
\Theta i j^{0 c}\left(\phi_{1}\right) & \Theta i j^{1 c}\left(\phi_{1}\right) & \Theta i j^{1 s}\left(\phi_{1}\right) & \ldots & \Theta i j^{M c}\left(\phi_{1}\right) & \Theta i j^{M s}\left(\phi_{1}\right) \\
\Theta i j^{0 c}\left(\phi_{2}\right) & \Theta i j^{1 c}\left(\phi_{2}\right) & \Theta i j^{1 s}\left(\phi_{2}\right) & \ldots & \Theta i j^{M c}\left(\phi_{2}\right) & \Theta i j^{M s}\left(\phi_{2}\right) \\
\Theta i j^{0 c}\left(\phi_{3}\right) & \Theta i j^{1 c}\left(\phi_{3}\right) & \Theta i j^{1 s}\left(\phi_{3}\right) & \ldots & \Theta i j^{M c}\left(\phi_{3}\right) & \Theta i j^{M s}\left(\phi_{3}\right) \\
\vdots & \vdots & \vdots & \ddots & \vdots & \vdots \\
\Theta i j^{0 c}\left(\phi_{2 M}\right) & \Theta i j^{1 c}\left(\phi_{2 M}\right) & \Theta i j^{1 s}\left(\phi_{2 M}\right) & \ldots & \Theta i j^{M c}\left(\phi_{2 M}\right) & \Theta i j^{M s}\left(\phi_{2 M}\right) \\
\Theta i j^{0 c}\left(\phi_{2 M+1}\right) & \Theta i j^{1 c}\left(\phi_{2 M+1}\right) & \Theta i j^{1 s}\left(\phi_{2 M+1}\right) & \ldots & \Theta i j^{M c}\left(\phi_{2 M+1}\right) & \Theta i j^{M s}\left(\phi_{2 M+1}\right)
\end{array}\right]}
\end{aligned}
$$

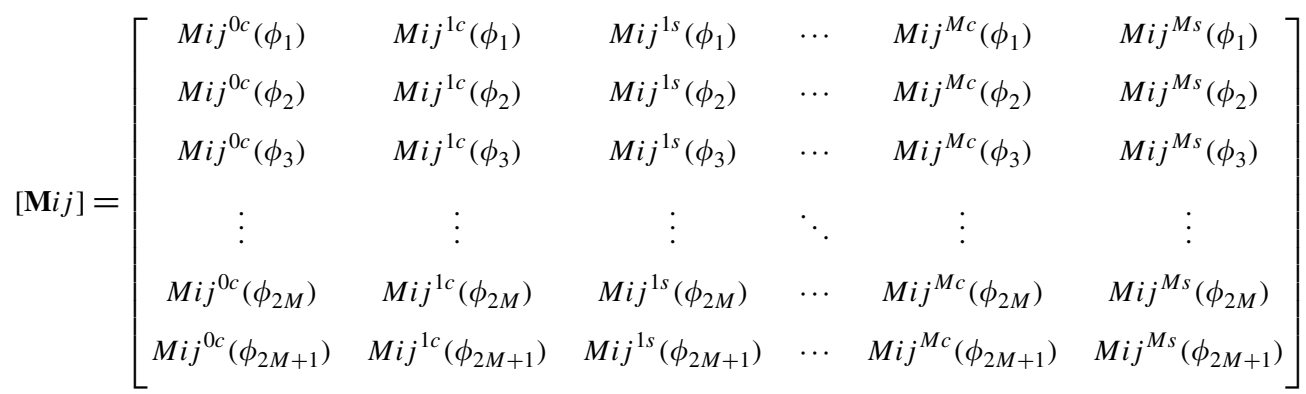

$$
\begin{aligned}
& {[\mathbf{V} i j]=\left[\begin{array}{cccccc}
V i j^{0 c}\left(\phi_{1}\right) & V i j^{1 c}\left(\phi_{1}\right) & V i j^{1 s}\left(\phi_{1}\right) & \ldots & V i j^{M c}\left(\phi_{1}\right) & V i j^{M s}\left(\phi_{1}\right) \\
V i j^{0 c}\left(\phi_{2}\right) & V i j^{1 c}\left(\phi_{2}\right) & V i j^{1 s}\left(\phi_{2}\right) & \ldots & V i j^{M c}\left(\phi_{2}\right) & V i j^{M s}\left(\phi_{2}\right) \\
V i j^{0 c}\left(\phi_{3}\right) & V i j^{1 c}\left(\phi_{3}\right) & V i j^{1 s}\left(\phi_{3}\right) & \ldots & V i j^{M c}\left(\phi_{3}\right) & V i j^{M s}\left(\phi_{3}\right) \\
\vdots & \vdots & \vdots & \ddots & \vdots & \vdots \\
V i j^{0 c}\left(\phi_{2 M}\right) & V i j^{1 c}\left(\phi_{2 M}\right) & V i j^{1 s}\left(\phi_{2 M}\right) & \ldots & V i j^{M c}\left(\phi_{2 M}\right) & V i j^{M s}\left(\phi_{2 M}\right) \\
V i j^{0 c}\left(\phi_{2 M+1}\right) & V i j^{1 c}\left(\phi_{2 M+1}\right) & V i j^{1 s}\left(\phi_{2 M+1}\right) & \ldots & V i j^{M c}\left(\phi_{2 M+1}\right) & V^{M s} j^{M s}\left(\phi_{2 M+1}\right)
\end{array}\right]}
\end{aligned}
$$




\section{J.-T. CHEN, C.-C. HSIAO AND S.-Y. LEU}

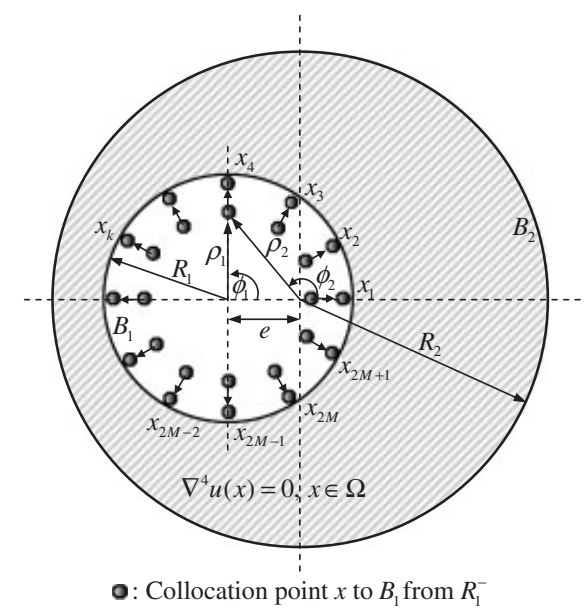

Figure 4. Sketch of the null-field points near the inner cylinder for the eccentric case.

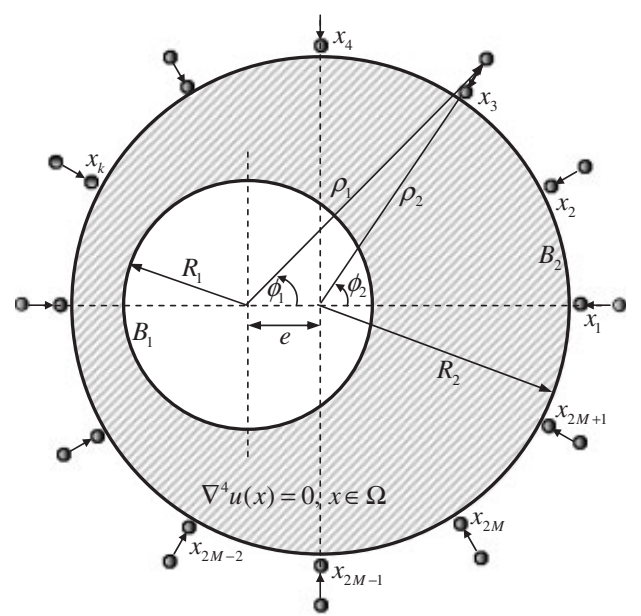

a: Collocation point $x$ to $B_{2}$ from $R_{2}^{+}$

Figure 5. Sketch of the null-field points near the outer cylinder for the eccentric case.

$$
\left[\mathbf{U} i j_{\theta}\right]=\left[\begin{array}{cccccc}
U i j_{\theta}^{0 c}\left(\phi_{1}\right) & U i j_{\theta}^{1 c}\left(\phi_{1}\right) & U i j_{\theta}^{1 s}\left(\phi_{1}\right) & \ldots & U i j_{\theta}^{M c}\left(\phi_{1}\right) & U i j_{\theta}^{M s}\left(\phi_{1}\right) \\
U i j_{\theta}^{0 c}\left(\phi_{2}\right) & U i j_{\theta}^{1 c}\left(\phi_{2}\right) & U i j_{\theta}^{1 s}\left(\phi_{2}\right) & \ldots & U i j_{\theta}^{M c}\left(\phi_{2}\right) & U i j_{\theta}^{M s}\left(\phi_{2}\right) \\
U i j_{\theta}^{0 c}\left(\phi_{3}\right) & U i j_{\theta}^{1 c}\left(\phi_{3}\right) & U i j_{\theta}^{1 s}\left(\phi_{3}\right) & \ldots & U i j_{\theta}^{M c}\left(\phi_{3}\right) & U i j_{\theta}^{M s}\left(\phi_{3}\right) \\
\vdots & \vdots & \vdots & \ddots & \vdots & \vdots \\
U i j_{\theta}^{0 c}\left(\phi_{2 M}\right) & U i j_{\theta}^{1 c}\left(\phi_{2 M}\right) & U i j_{\theta}^{1 s}\left(\phi_{2 M}\right) & \ldots & U i j_{\theta}^{M c}\left(\phi_{2 M}\right) & U i j_{\theta}^{M s}\left(\phi_{2 M}\right) \\
U i j_{\theta}^{0 c}\left(\phi_{2 M+1}\right) & U i j_{\theta}^{1 c}\left(\phi_{2 M+1}\right) & U i j_{\theta}^{1 s}\left(\phi_{2 M+1}\right) & \ldots & U i j_{\theta}^{M c}\left(\phi_{2 M+1}\right) & U i j_{\theta}^{M s}\left(\phi_{2 M+1}\right)
\end{array}\right]
$$


A NEW METHOD FOR STOKES PROBLEMS WITH CIRCULAR BOUNDARIES

$$
\begin{aligned}
& {\left[\Theta i j_{\theta}\right]=\left[\begin{array}{cccccc}
\Theta i j_{\theta}^{0 c}\left(\phi_{1}\right) & \Theta i j_{\theta}^{1 c}\left(\phi_{1}\right) & \Theta i j_{\theta}^{1 s}\left(\phi_{1}\right) & \ldots & \Theta i j_{\theta}^{M c}\left(\phi_{1}\right) & \Theta i j_{\theta}^{M s}\left(\phi_{1}\right) \\
\Theta i j_{\theta}^{0 c}\left(\phi_{2}\right) & \Theta i j_{\theta}^{1 c}\left(\phi_{2}\right) & \Theta i j_{\theta}^{1 s}\left(\phi_{2}\right) & \ldots & \Theta i j_{\theta}^{M c}\left(\phi_{2}\right) & \Theta i j_{\theta}^{M s}\left(\phi_{2}\right) \\
\Theta i j_{\theta}^{0 c}\left(\phi_{3}\right) & \Theta i j_{\theta}^{1 c}\left(\phi_{3}\right) & \Theta i j_{\theta}^{1 s}\left(\phi_{3}\right) & \ldots & \Theta i j_{\theta}^{M c}\left(\phi_{3}\right) & \Theta i j_{\theta}^{M s}\left(\phi_{3}\right) \\
\vdots & \vdots & \vdots & \ddots & \vdots & \vdots \\
\Theta i j_{\theta}^{0 c}\left(\phi_{2 M}\right) & \Theta i j_{\theta}^{1 c}\left(\phi_{2 M}\right) & \Theta i j_{\theta}^{1 s}\left(\phi_{2 M}\right) & \ldots & \Theta i j_{\theta}^{M c}\left(\phi_{2 M}\right) & \Theta i j_{\theta}^{M s}\left(\phi_{2 M}\right) \\
\Theta i j_{\theta}^{0 c}\left(\phi_{2 M+1}\right) & \Theta i j_{\theta}^{1 c}\left(\phi_{2 M+1}\right) & \Theta i j_{\theta}^{1 s}\left(\phi_{2 M+1}\right) & \cdots & \Theta i j_{\theta}^{M c}\left(\phi_{2 M+1}\right) & \Theta i j_{\theta}^{M s}\left(\phi_{2 M+1}\right)
\end{array}\right]}
\end{aligned}
$$

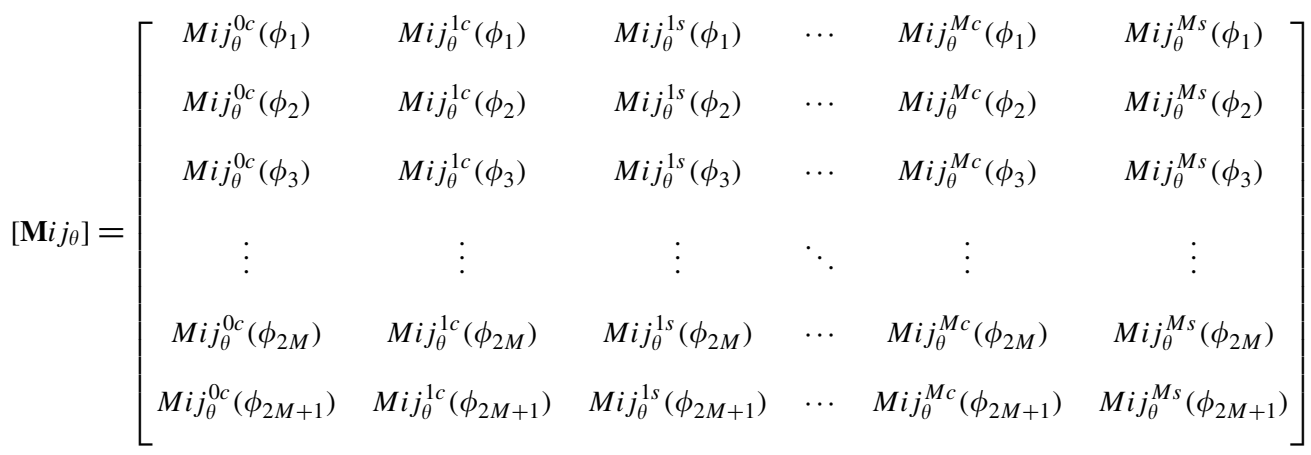

$$
\left[\mathbf{V i j _ { \theta } ]}=\left[\begin{array}{cccccc}
V i j_{\theta}^{0 c}\left(\phi_{1}\right) & V i j_{\theta}^{1 c}\left(\phi_{1}\right) & V i j_{\theta}^{1 s}\left(\phi_{1}\right) & \ldots & V i j_{\theta}^{M c}\left(\phi_{1}\right) & V i j_{\theta}^{M s}\left(\phi_{1}\right) \\
V i j_{\theta}^{0 c}\left(\phi_{2}\right) & V i j_{\theta}^{1 c}\left(\phi_{2}\right) & V i j_{\theta}^{1 s}\left(\phi_{2}\right) & \cdots & V i j_{\theta}^{M c}\left(\phi_{2}\right) & V i j_{\theta}^{M s}\left(\phi_{2}\right) \\
V i j_{\theta}^{0 c}\left(\phi_{3}\right) & V i j_{\theta}^{1 c}\left(\phi_{3}\right) & V i j_{\theta}^{1 s}\left(\phi_{3}\right) & \ldots & V i j_{\theta}^{M c}\left(\phi_{3}\right) & V i j_{\theta}^{M s}\left(\phi_{3}\right) \\
\vdots & \vdots & \vdots & \ddots & \vdots \\
V i j_{\theta}^{0 c}\left(\phi_{2 M}\right) & V i j_{\theta}^{1 c}\left(\phi_{2 M}\right) & V i j_{\theta}^{1 s}\left(\phi_{2 M}\right) & \ldots & V i j_{\theta}^{M c}\left(\phi_{2 M}\right) & V i j_{\theta}^{M s}\left(\phi_{2 M}\right) \\
V i j_{\theta}^{0 c}\left(\phi_{2 M+1}\right) & V i j_{\theta}^{1 c}\left(\phi_{2 M+1}\right) & V i j_{\theta}^{1 s}\left(\phi_{2 M+1}\right) & \cdots & V i j_{\theta}^{M c}\left(\phi_{2 M+1}\right) & V i j_{\theta}^{M s}\left(\phi_{2 M+1}\right)
\end{array}\right]\right.
$$

where $\phi_{k}(k=1,2,3, \ldots, 2 M+1)$ is the $k$ th collocation angle of the collocation points on each boundary and the elements of the sub-matrix are defined as follows:

$$
\begin{aligned}
& U i j^{n c}\left(\phi_{k}\right)=\int_{B_{j}} U\left(s, x_{k}\right) \cos \left(n \theta_{j}\right) R_{j} \mathrm{~d} \theta_{j}, \quad n=0,1,2,3, \ldots, M \\
& U i j^{n s}\left(\phi_{k}\right)=\int_{B_{j}} U\left(s, x_{k}\right) \sin \left(n \theta_{j}\right) R_{j} \mathrm{~d} \theta_{j}, \quad n=1,2,3, \ldots, M
\end{aligned}
$$




$$
\begin{aligned}
& \Theta i j^{n c}\left(\phi_{k}\right)=\int_{B_{j}} \Theta\left(s, x_{k}\right) \cos \left(n \theta_{j}\right) R_{j} \mathrm{~d} \theta_{j}, \quad n=0,1,2,3, \ldots, M \\
& \Theta i j^{n s}\left(\phi_{k}\right)=\int_{B_{j}} \Theta\left(s, x_{k}\right) \sin \left(n \theta_{j}\right) R_{j} \mathrm{~d} \theta_{j}, \quad n=1,2,3, \ldots, M \\
& \operatorname{Mij}^{n c}\left(\phi_{k}\right)=\int_{B_{j}} M\left(s, x_{k}\right) \cos \left(n \theta_{j}\right) R_{j} \mathrm{~d} \theta_{j}, \quad n=0,1,2,3, \ldots, M \\
& \operatorname{Mij}^{n s}\left(\phi_{k}\right)=\int_{B_{j}} M\left(s, x_{k}\right) \sin \left(n \theta_{j}\right) R_{j} \mathrm{~d} \theta_{j}, \quad n=1,2,3, \ldots, M \\
& V i j^{n c}\left(\phi_{k}\right)=\int_{B_{j}} V\left(s, x_{k}\right) \cos \left(n \theta_{j}\right) R_{j} \mathrm{~d} \theta_{j}, \quad n=0,1,2,3, \ldots, M \\
& V i j^{n s}\left(\phi_{k}\right)=\int_{B_{j}} V\left(s, x_{k}\right) \sin \left(n \theta_{j}\right) R_{j} \mathrm{~d} \theta_{j}, \quad n=1,2,3, \ldots, M \\
& U i j_{\theta}^{n c}\left(\phi_{k}\right)=\int_{B_{j}} U_{\theta}\left(s, x_{k}\right) \cos \left(n \theta_{j}\right) R_{j} \mathrm{~d} \theta_{j}, \quad n=0,1,2,3, \ldots, M \\
& U i j_{\theta}^{n s}\left(\phi_{k}\right)=\int_{B_{j}} U_{\theta}\left(s, x_{k}\right) \sin \left(n \theta_{j}\right) R_{j} \mathrm{~d} \theta_{j}, \quad n=1,2,3, \ldots, M \\
& \Theta i j_{\theta}^{n c}\left(\phi_{k}\right)=\int_{B_{j}} \Theta_{\theta}\left(s, x_{k}\right) \cos \left(n \theta_{j}\right) R_{j} \mathrm{~d} \theta_{j}, \quad n=0,1,2,3, \ldots, M \\
& \Theta i j_{\theta}^{n s}\left(\phi_{k}\right)=\int_{B_{j}} \Theta_{\theta}\left(s, x_{k}\right) \sin \left(n \theta_{j}\right) R_{j} \mathrm{~d} \theta_{j}, \quad n=1,2,3, \ldots, M \\
& \operatorname{Mij}_{\theta}^{n c}\left(\phi_{k}\right)=\int_{B_{j}} M_{\theta}\left(s, x_{k}\right) \cos \left(n \theta_{j}\right) R_{j} \mathrm{~d} \theta_{j}, \quad n=0,1,2,3, \ldots, M \\
& M i j_{\theta}^{n s}\left(\phi_{k}\right)=\int_{B_{j}} M_{\theta}\left(s, x_{k}\right) \sin \left(n \theta_{j}\right) R_{j} \mathrm{~d} \theta_{j}, \quad n=1,2,3, \ldots, M \\
& \operatorname{Vij}_{\theta}^{n c}\left(\phi_{k}\right)=\int_{B_{j}} V_{\theta}\left(s, x_{k}\right) \cos \left(n \theta_{j}\right) R_{j} \mathrm{~d} \theta_{j}, \quad n=0,1,2,3, \ldots, M \\
& V i j_{\theta}^{n s}\left(\phi_{k}\right)=\int_{B_{j}} V_{\theta}\left(s, x_{k}\right) \sin \left(n \theta_{j}\right) R_{j} \mathrm{~d} \theta_{j}, \quad n=1,2,3, \ldots, M
\end{aligned}
$$




\section{A NEW METHOD FOR STOKES PROBLEMS WITH CIRCULAR BOUNDARIES}

where the interior degenerate kernels are used for $i=1$ and $j=1,2$; the exterior degenerate kernels are used for $i=2$ and $j=1,2$. However, the stream function on the boundary of inner rotating cylinder is an unknown constant $u_{1}[13,14]$ for the viscous flow problems. In other words, one more unknown degree of freedom is introduced in the real implementation. Therefore, an extra constraint is required to uniquely solve the problem. The additional equation is obtained on the physical view that the pressure is periodic in $2 \pi$ around the inner rotating cylinder. According to the Stokes equation of motion and $\nabla^{2} u=\omega$, the pressure $P$ and vorticity $\omega$ satisfy the Cauchy-Riemann equation, the condition for periodicity in $P$, namely

$$
\int_{B_{1}} \frac{\partial P}{\partial t} \mathrm{~d} B_{1}=0
$$

becomes

$$
\int_{B_{1}} \frac{\partial \omega}{\partial n} \mathrm{~d} B_{1}=\int_{B_{1}} \omega_{n} \mathrm{~d} B_{1}=0
$$

where $\omega_{n}$ is the normal derivative of vorticity, $t$ and $n$ are tangent and normal vectors on the boundary for the Cauchy-Riemann relation. If $u$ is solved, the vorticity can be determined by $\omega=\nabla^{2} u$ in the post-processing using Equation (18). Therefore, $\omega_{n}$ can be obtained by taking normal derivative with respect to $\omega(x)$ in Equation (18)

$$
\begin{aligned}
\omega_{n}= & \frac{1}{8 \pi} \sum_{j=1}^{N_{\mathrm{C}}} \int_{B_{j}}\left\{-U_{\nabla^{2}, n}(s, x) v_{j}(s)+\Theta_{\nabla^{2}, n}(s, x) m_{j}(s)\right. \\
& \left.-M_{\nabla^{2}, n}(s, x) \theta_{j}(s)+V_{\nabla^{2}, n}(s, x) u_{j}(s)\right\} \mathrm{d} B_{j}(s)
\end{aligned}
$$

in which $U_{\nabla^{2}, n}(s, x), \Theta_{\nabla^{2}, n}(s, x), M_{\nabla^{2}, n}(s, x)$ and $V_{\nabla^{2}, n}(s, x)$ are the normal derivatives of Laplacian of the degenerate kernels $U(s, x), \Theta(s, x), M(s, x)$ and $V(s, x)$, respectively, which are listed in Appendix A, $N_{C}$ is the number of circular boundaries. By substituting Equation (65) into Equation (64), we have the constraint equation

$$
\begin{aligned}
& \int_{B_{1}}\left\{\sum _ { j = 1 } ^ { N _ { \mathrm { C } } } \int _ { B _ { j } } \left[-U_{\nabla^{2}, n}(s, x) v_{j}(s)+\Theta_{\nabla^{2}, n}(s, x) m_{j}(s)\right.\right. \\
& \left.\left.\quad-M_{\nabla^{2}, n}(s, x) \theta_{j}(s)+V_{\nabla^{2}, n}(s, x) u_{j}(s)\right] \mathrm{d} B_{j}(s)\right\} \mathrm{d} B_{1}(x)=0
\end{aligned}
$$

Equation (66) indicates that the constraint is composed of double boundary integrals. It is noted that the point $x$ in the first boundary integral is located by approaching $x$ from the domain to $R_{1}^{+}$as shown in Figure 6 . For the double integration of the same inner boundaries $\int_{B_{1}} \int_{B_{1}}$, the analytical integration can be obtained by using the orthogonal property of Fourier bases. For the double integration on different boundaries $\int_{B_{1}} \int_{B_{2}}$, trapezoid integral is used as follows:

$$
\int_{0}^{2 \pi} f(\phi) \mathrm{d} \phi=\sum_{k=1}^{N} \frac{2 \pi}{N} f\left(\phi_{k}\right)
$$




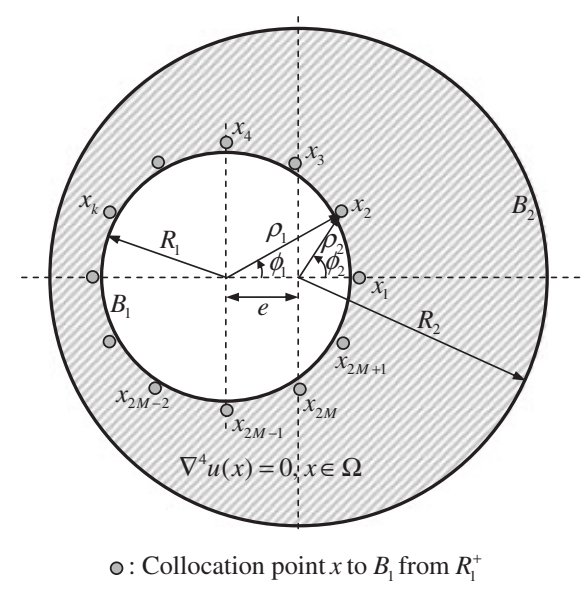

Figure 6. Collocation method for the constraint equation.

where the outer boundary is uniformly divided into $N$ segments. By matching the boundary conditions at the $2 M+1$ collocation points on each boundary and rearranging the known and unknown sets, the linear algebraic system Equation (39) is reformulated to

$$
\left[\begin{array}{ccccc}
\mathbf{U} 11 & \boldsymbol{\Theta} 11 & \mathbf{U} 12 & \boldsymbol{\Theta} 12^{2} & \mathbf{V} 11 \\
\mathbf{U} 11_{\theta} & \boldsymbol{\Theta} 11_{\theta} & \mathbf{U} 12_{\theta} & \boldsymbol{\Theta} 12_{\theta} & \mathbf{V} 11_{\theta} \\
\mathbf{U} 21 & \boldsymbol{\Theta} 21 & \mathbf{U} 22 & \boldsymbol{\Theta} 22 & \mathbf{V} 21 \\
\mathbf{U} 21_{\theta} & \boldsymbol{\Theta} 21_{\theta} & \mathbf{U} 22_{\theta} & \boldsymbol{\Theta} 22_{\theta} & \mathbf{V} 21_{\theta} \\
\mathbf{U} 11_{\nabla^{2}, n} & \boldsymbol{\Theta} 11_{\nabla^{2}, n} & \mathbf{U} 12_{\nabla^{2}, n} & \boldsymbol{\Theta} 12_{\nabla^{2}, n} & \mathbf{V} 11_{\nabla^{2}, n}
\end{array}\right]\left\{\begin{array}{c}
\mathbf{v}_{1} \\
\mathbf{m}_{1} \\
\mathbf{v} 2 \\
\mathbf{m}_{2} \\
u_{1}
\end{array}\right\}=\theta_{1}\left\{\begin{array}{c}
\mathbf{M} 11 \\
\mathbf{M} 11_{\theta} \\
\mathbf{M} 21 \\
\mathbf{M} 21_{\theta} \\
\mathbf{M} 11_{\nabla^{2}, n}
\end{array}\right\}
$$

where $\theta_{1}=\omega_{1} r_{1}$ due to the rotation of inner cylinder $[13,14]$. It is noted that $[\mathbf{V 1 2}],\left[\mathbf{V} 12_{\theta}\right]$, [V22], [V22 $\left.{ }_{\theta}\right],\left[\mathbf{V} 12_{\nabla^{2}, n}\right],[\mathbf{M} 12],\left[\mathbf{M} 12_{\theta}\right],[\mathbf{M} 22],\left[\mathbf{M} 22_{\theta}\right]$ and $\left[\mathbf{M} 12_{\nabla^{2}, n}\right]$ disappear since the outer cylinder is stationary $\left(u_{2}=0\right.$ and $\left.\theta_{2}=0\right)$. The sub-matrices $\left[\mathbf{U} 11_{\nabla^{2}, n}\right],\left[\boldsymbol{\Theta} 11_{\nabla^{2}, n}\right],\left[\mathbf{U} 12_{\nabla^{2}, n}\right]$ and $\left[\Theta 12_{\nabla^{2}, n}\right]$ with a dimension of one by $(2 M+1)$ are shown below:

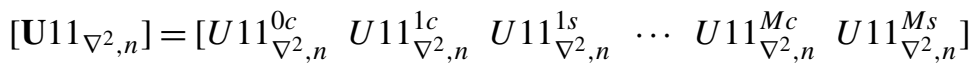

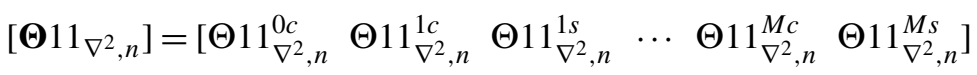

$$
\begin{aligned}
& {\left[\begin{array}{llllll}
\mathbf{U} 12_{\nabla^{2}, n} & ]=\left[\begin{array}{lllll}
U 12_{\nabla^{2}, n}^{0 c} & U 12_{\nabla^{2}, n}^{1 c} & U 12_{\nabla^{2}, n}^{1 s} & \cdots & U 12_{\nabla^{2}, n}^{M c}
\end{array} \quad U 12_{\nabla^{2}, n}^{M s}\right.
\end{array}\right]}
\end{aligned}
$$

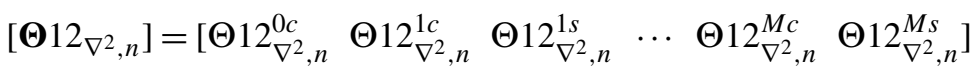




\section{A NEW METHOD FOR STOKES PROBLEMS WITH CIRCULAR BOUNDARIES}

where each element of $\left[\mathbf{U} 11_{\nabla^{2}, n}\right],\left[\Theta 11_{\nabla^{2}, n}\right],\left[\mathbf{U} 12_{\nabla^{2}, n}\right]$ and $\left[\boldsymbol{\Theta} 12_{\nabla^{2}, n}\right]$ are defined as shown below:

$$
\begin{aligned}
& U 11_{\nabla^{2}, n}^{n c}=\int_{B_{1}} \int_{B_{1}} U_{\nabla^{2}, n}^{\mathrm{E}}(s, x) \cos \left(n \theta_{1}\right) \mathrm{d} B_{1}(s) \mathrm{d} B(x)_{1}, \quad n=0,1,2,3, \ldots, M \\
& U 11_{\nabla^{2}, n}^{n s}=\int_{B_{1}} \int_{B_{1}} U_{\nabla^{2}, n}^{\mathrm{E}}(s, x) \sin \left(n \theta_{1}\right) \mathrm{d} B_{1}(s) \mathrm{d} B_{1}(x), \quad n=1,2,3, \ldots, M \\
& \Theta 11_{\nabla^{2}, n}^{n c}=\int_{B_{1}} \int_{B_{1}} \Theta_{\nabla^{2}, n}^{\mathrm{E}}(s, x) \cos \left(n \theta_{1}\right) \mathrm{d} B_{1}(s) \mathrm{d} B_{1}(x), \quad n=0,1,2,3, \ldots, M \\
& \Theta 11_{\nabla^{2}, n}^{n s}=\int_{B_{1}} \int_{B_{1}} \Theta_{\nabla^{2}, n}^{\mathrm{E}}(s, x) \sin \left(n \theta_{1}\right) \mathrm{d} B_{1}(s) \mathrm{d} B_{1}(x), \quad n=1,2,3, \ldots, M \\
& U 12_{\nabla^{2}, n}^{n c}=\int_{B_{1}} \int_{B_{2}} U_{\nabla^{2}, n}^{\mathrm{I}}\left(s, x_{k}\right) \cos \left(n \theta_{2}\right) \mathrm{d} B_{2}(s) \mathrm{d} B_{1}(x) \\
& =\sum_{k=1}^{N} \frac{2 \pi}{N} \int_{B_{2}} U_{\nabla^{2}, n}^{\mathrm{I}}\left(s, x_{k}\right) \cos \left(n \theta_{2}\right) \mathrm{d} B_{2}(s), \quad n=0,1,2,3, \ldots, M \\
& U 12_{\nabla^{2}, n}^{n s}=\int_{B_{1}} \int_{B_{2}} U_{\nabla^{2}, n}^{\mathrm{I}}\left(s, x_{k}\right) \sin \left(n \theta_{2}\right) \mathrm{d} B_{2}(s) \mathrm{d} B_{1}(x) \\
& =\sum_{k=1}^{N} \frac{2 \pi}{N} \int_{B_{2}} U_{\nabla^{2}, n}^{\mathrm{I}}\left(s, x_{k}\right) \sin \left(n \theta_{2}\right) \mathrm{d} B_{2}(s), \quad n=1,2,3, \ldots, M \\
& \Theta 12_{\nabla^{2}, n}^{n c}=\int_{B_{1}} \int_{B_{2}} \Theta_{\nabla^{2}, n}^{\mathrm{I}}\left(s, x_{k}\right) \cos \left(n \theta_{2}\right) \mathrm{d} B_{2}(s) \mathrm{d} B_{1}(x) \\
& =\sum_{k=1}^{N} \frac{2 \pi}{N} \int_{B_{2}} \Theta_{\nabla^{2}, n}^{\mathrm{I}}\left(s, x_{k}\right) \cos \left(n \theta_{2}\right) \mathrm{d} B_{2}(s), \quad n=0,1,2,3, \ldots, M \\
& \Theta 12_{\nabla^{2}, n}^{n s}=\int_{B_{1}} \int_{B_{2}} \Theta_{\nabla^{2}, n}^{\mathrm{I}}\left(s, x_{k}\right) \sin \left(n \theta_{2}\right) \mathrm{d} B_{2}(s) \mathrm{d} B_{1}(x) \\
& =\sum_{k=1}^{N} \frac{2 \pi}{N} \int_{B_{2}} \Theta_{\nabla^{2}, n}^{\mathrm{I}}\left(s, x_{k}\right) \sin \left(n \theta_{2}\right) \mathrm{d} B_{2}(s), \quad n=1,2,3, \ldots, M
\end{aligned}
$$


where $x_{k}$ is the sampling point. The elements of $\left[\mathbf{M} 11_{\nabla^{2}, n}\right]$ and $\left[\mathbf{V} 11_{\nabla^{2}, n}\right]$ with a dimension of one by one are defined as follows:

$$
\begin{aligned}
M 11_{\nabla^{2}, n} & =\int_{B_{1}} \int_{B_{1}} M_{\nabla^{2}, n}^{\mathrm{E}}(s, x) 1 \mathrm{~d} B_{1}(s) \mathrm{d} B_{1}(x) \\
V 11_{\nabla^{2}, n} & =\int_{B_{1}} \int_{B_{1}} V_{\nabla^{2}, n}^{\mathrm{E}}(s, x) 1 \mathrm{~d} B_{1}(s) \mathrm{d} B_{1}(x)
\end{aligned}
$$

The unknown Fourier coefficients and the unknown stream function on the inner rotating cylinder can be obtained at the same time by solving the linear algebraic augmented system of Equation (68). After determining the unknown Fourier coefficients, the interior potential can be obtained by using the BIE for the domain point. The vorticity in the post-processing can be obtained by using the following equation:

$$
\begin{aligned}
\nabla^{2} u(x)= & \omega(x)=\frac{1}{8 \pi} \sum_{j=1}^{N_{\mathrm{C}}}\left\{-\int_{B_{j}} U_{\nabla^{2}}(s, x) v_{j}(s) \mathrm{d} B_{j}(s)+\int_{B_{j}} \Theta_{\nabla^{2}}(s, x) v_{j}(s) \mathrm{d} B_{j}(s)\right. \\
& \left.-\int_{B_{j}} M_{\nabla^{2}}(s, x) v_{j}(s) \mathrm{d} B_{j}(s)+\int_{B_{j}} V_{\nabla^{2}}(s, x) v_{j}(s) \mathrm{d} B_{j}(s)\right\}, \quad x \in \Omega
\end{aligned}
$$

where $N_{\mathrm{C}}$ is the number of circular boundaries.

\subsection{Indirect formulation}

By using the indirect formulation of Equations (28)-(29) and collocating to the boundaries from $R^{+}$and $R^{-}$for the inner and outer boundaries, respectively, the linear algebraic system is obtained as follows:

$$
\left[\begin{array}{cccc}
\mathbf{U} 11 & \boldsymbol{\Theta} 11 & \mathbf{U} 12 & \boldsymbol{\Theta} 12 \\
\mathbf{U} 11_{\boldsymbol{\theta}} & \boldsymbol{\Theta} 11_{\boldsymbol{\theta}} & \mathbf{U} 12_{\boldsymbol{\theta}} & \boldsymbol{\Theta} 12_{\boldsymbol{\theta}} \\
\mathbf{U} 21 & \boldsymbol{\Theta} 21 & \mathbf{U} 22 & \boldsymbol{\Theta} 22 \\
\mathbf{U} 21_{\boldsymbol{\theta}} & \boldsymbol{\Theta} 21_{\boldsymbol{\theta}} & \mathbf{U} 22_{\boldsymbol{\theta}} & \boldsymbol{\Theta} 22_{\boldsymbol{\theta}}
\end{array}\right]\left\{\begin{array}{l}
\boldsymbol{\Phi}_{1} \\
\boldsymbol{\Psi}_{1} \\
\boldsymbol{\Phi}_{2} \\
\boldsymbol{\Psi}_{2}
\end{array}\right\}=\left\{\begin{array}{l}
u_{1} \\
\theta_{1} \\
u_{2} \\
\theta_{2}
\end{array}\right\}
$$

where $\boldsymbol{\Phi}_{1}, \boldsymbol{\Psi}_{1}, \boldsymbol{\Phi}_{2}$ and $\boldsymbol{\Psi}_{2}$ are the column vectors of Fourier coefficients for the fictitious boundary distributions of $\boldsymbol{\Phi}$ and $\boldsymbol{\Psi} ; u_{1}, \theta_{1}, u_{2}$ and $\theta_{2}$ are the given boundary conditions. The sub-matrices $[\mathbf{U} i j],[\Theta i j],\left[\mathbf{U} i j_{\boldsymbol{\theta}}\right]$ and $\left[\boldsymbol{\Theta} i j_{\boldsymbol{\theta}}\right](i=1,2$ and $j=1,2)$ of the influence matrix are the same as Equations (40) and (44)-(45). The elements of the sub-matrices are defined as follows:

$$
\begin{aligned}
& U i j^{n c}\left(\phi_{k}\right)=\int_{B_{j}} U\left(s, x_{k}\right) \cos \left(n \theta_{j}\right) R_{j} \mathrm{~d} \theta_{j}, \quad n=0,1,2,3, \ldots, M \\
& U i j^{n s}\left(\phi_{k}\right)=\int_{B_{j}} U\left(s, x_{k}\right) \sin \left(n \theta_{j}\right) R_{j} \mathrm{~d} \theta_{j}, \quad n=1,2,3, \ldots, M
\end{aligned}
$$


A NEW METHOD FOR STOKES PROBLEMS WITH CIRCULAR BOUNDARIES

$$
\begin{aligned}
& \Theta i j^{n c}\left(\phi_{k}\right)=\int_{B_{j}} \Theta\left(s, x_{k}\right) \cos \left(n \theta_{j}\right) R_{j} \mathrm{~d} \theta_{j}, \quad n=0,1,2,3, \ldots, M \\
& \Theta i j^{n s}\left(\phi_{k}\right)=\int_{B_{j}} \Theta\left(s, x_{k}\right) \sin \left(n \theta_{j}\right) R_{j} \mathrm{~d} \theta_{j}, \quad n=1,2,3, \ldots, M \\
& U i j_{\theta}^{n c}\left(\phi_{k}\right)=\int_{B_{j}} U_{\theta}\left(s, x_{k}\right) \cos \left(n \theta_{j}\right) R_{j} \mathrm{~d} \theta_{j}, \quad n=0,1,2,3, \ldots, M \\
& U i j_{\theta}^{n s}\left(\phi_{k}\right)=\int_{B_{j}} U_{\theta}\left(s, x_{k}\right) \sin \left(n \theta_{j}\right) R_{j} \mathrm{~d} \theta_{j}, \quad n=1,2,3, \ldots, M \\
& \Theta i j_{\theta}^{n c}(\phi k)=\int_{B_{j}} \Theta_{\theta}\left(s, x_{k}\right) \cos \left(n \theta_{j}\right) R_{j} \mathrm{~d} \theta_{j}, \quad n=0,1,2,3, \ldots, M \\
& \Theta i j_{\theta}^{n s}(\phi k)=\int_{B_{j}} \Theta_{\theta}\left(s, x_{k}\right) \sin \left(n \theta_{j}\right) R_{j} \mathrm{~d} \theta_{j}, \quad n=1,2,3, \ldots, M
\end{aligned}
$$

where $j=1$ and $i=1,2$, the exterior degenerate kernels are used; $j=2$ and $i=1,2$, the interior degenerate kernels are used. However, $u_{1}$ is an unknown constant along the inner cylinder as explained in the direct BIEM, one more constraint equation is needed and Equation (64) is considered again as follows:

$$
\int_{B_{1}} \frac{\partial \omega}{\partial n} \mathrm{~d} B_{1}=\int_{B_{1}} \omega_{n} \mathrm{~d} B_{1}=0
$$

By substituting Equation (32) into Equation (93), we have

$$
\int_{B_{1}} \sum_{j=1}^{N_{\mathrm{C}}}\left\{\int_{B_{j}} U_{\nabla^{2}, n}(s, x) \Phi_{j}(s) \mathrm{d} B_{j}(s)+\int_{B_{j}} \Theta_{\nabla^{2}, n}(s, x) \Psi_{j}(s) \mathrm{d} B_{j}(s)\right\} \mathrm{d} B_{1}(x)=0
$$

Therefore, the linear algebraic system (84) can be reformulated as shown below:

$$
\left[\begin{array}{ccccc}
\mathbf{U} 11 & \boldsymbol{\Theta} 11 & \mathbf{U} 12 & \boldsymbol{\Theta} 12 & -1 \\
\mathbf{U} 11_{\boldsymbol{\theta}} & \boldsymbol{\Theta} 11_{\boldsymbol{\theta}} & \mathbf{U} 12_{\boldsymbol{\theta}} & \boldsymbol{\Theta} 12_{\boldsymbol{\theta}} & 0 \\
\mathbf{U} 21 & \boldsymbol{\Theta} 21 & \mathbf{U} 22 & \boldsymbol{\Theta} 22 & 0 \\
\mathbf{U} 21_{\boldsymbol{\theta}} & \boldsymbol{\Theta} 21_{\boldsymbol{\theta}} & \mathbf{U} 22_{\boldsymbol{\theta}} & \boldsymbol{\Theta} 22_{\boldsymbol{\theta}} & 0 \\
\mathbf{U} 11_{\nabla^{2}, n} & \boldsymbol{\Theta} 11_{\nabla^{2}, n} & \mathbf{U} 12_{\nabla^{2}, n} & \boldsymbol{\Theta} 12_{\nabla^{2}, n} & 0
\end{array}\right]\left\{\begin{array}{c}
\boldsymbol{\Phi}_{1} \\
\boldsymbol{\Psi}_{1} \\
\boldsymbol{\Phi}_{2} \\
\boldsymbol{\Psi}_{2} \\
u_{1}
\end{array}\right\}=\left\{\begin{array}{c}
0 \\
\theta_{1} \\
u_{2} \\
\theta_{2} \\
0
\end{array}\right\}
$$

The sub-matrices $\left[\mathbf{U} 11_{\nabla^{2}, n}\right],\left[\boldsymbol{\Theta} 11_{\nabla^{2}, n}\right]\left[\mathbf{U} 12_{\nabla^{2}, n}\right],\left[\boldsymbol{\Theta} 12_{\nabla^{2}, n}\right]$ with a dimension of one by $(2 M+1)$, respectively, are the same as Equations (69)-(72). The unknown Fourier coefficients and the unknown stream function along the inner rotating cylinder can be obtained at the same time by solving the linear algebraic augmented system of Equation (95). After determining the unknown Fourier coefficients, the interior potential can be obtained by using the BIE for the domain point 


\section{J.-T. CHEN, C.-C. HSIAO AND S.-Y. LEU}

of Equation (28). The vorticity in the post-processing can be obtained by using the following equation:

$$
\omega(x)=\sum_{j=1}^{N_{\mathrm{C}}}\left\{\int_{B_{j}} U_{\nabla^{2}}(s, x) \Phi_{j}(s) \mathrm{d} B_{j}(s)+\int_{B_{j}} \Theta_{\nabla^{2}}(s, x) \Psi_{j}(s) \mathrm{d} B_{j}(s)\right\}, \quad x \in \Omega
$$

where $N_{\mathrm{C}}$ is the number of circular boundaries.

\section{NUMERICAL EXAMPLES}

\subsection{Eccentric case: a doubly-connected domain}

Two approaches, direct BIEM and indirect BIEM, are presented to solve the flow between eccentric cylinders. The inner cylinder rotates with a constant angular velocity and the outer one is stationary as shown in Figure 7. The following parameters are defined: $r_{1}=0.5$, radius of inner cylinder; $r_{2}=1$, radius of outer cylinder; $c=r_{2}-r_{1}$, the clearance; $\varepsilon=e / c$, the eccentricity; $e$, separation of centers of cylinders; $\omega_{1}=1$ for the anticlockwise angular velocity of inner cylinder.

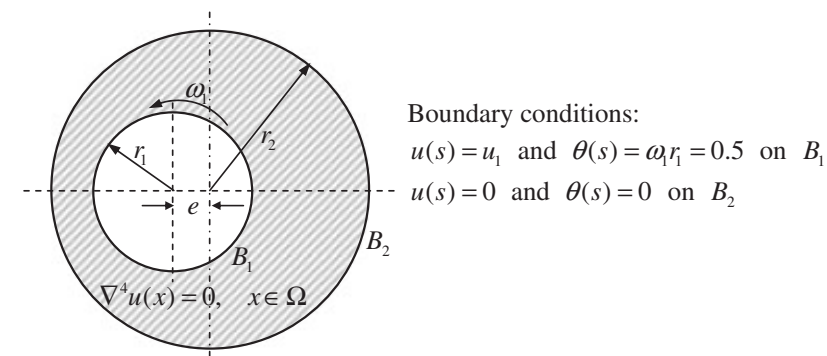

Figure 7. The flow between eccentric cylinders.

Table I. Comparison of analytical and numerical results of $u_{1}$ for the eccentric bearing.

\begin{tabular}{|c|c|c|c|c|c|c|c|}
\hline \multirow[b]{2}{*}{$\varepsilon$} & \multicolumn{4}{|c|}{ Kelmanson and Ingham $[13,14]$} & \multirow[b]{2}{*}{$\begin{array}{l}\text { Analytical } \\
\text { solution }\end{array}$} & \multirow[b]{2}{*}{$\begin{array}{l}\text { Present method } \\
\text { (Direct BIEM) }\end{array}$} & \multirow[b]{2}{*}{$\begin{array}{l}\text { Present method } \\
\text { (Indirect BIEM) }\end{array}$} \\
\hline & $n=80$ & $n=160$ & $n=320$ & $\underset{n \rightarrow \infty}{\operatorname{Limit}}$ & & & \\
\hline 0.0 & 0.1066 & 0.1062 & 0.1061 & 0.1061 & 0.1060 & $0.1060(N=5)$ & $0.1060(N=5)$ \\
\hline 0.1 & 0.1052 & 0.1048 & 0.1047 & 0.1047 & 0.1046 & $0.1046(N=7)$ & $0.1046(N=7)$ \\
\hline 0.2 & 0.1011 & 0.1006 & 0.1005 & 0.1005 & 0.1005 & $0.1005(N=7)$ & $0.1005(N=7)$ \\
\hline 0.3 & 0.0944 & 0.0939 & 0.0938 & 0.0938 & 0.0938 & $0.0938(N=7)$ & $0.0938(N=7)$ \\
\hline 0.4 & 0.0854 & 0.0850 & 0.0848 & 0.0846 & 0.0848 & $0.0848(N=9)$ & $0.0848(N=9)$ \\
\hline 0.5 & 0.0748 & 0.0740 & 0.0739 & 0.0739 & 0.0738 & $0.0738(N=11)$ & $0.0738(N=11)$ \\
\hline 0.6 & 0.0622 & 0.0615 & 0.0613 & 0.0612 & 0.0611 & $0.0611(N=17)$ & $0.0611 \quad(N=17)$ \\
\hline 0.7 & 0.0484 & 0.0477 & 0.0474 & 0.0472 & 0.0472 & $0.0472(N=17)$ & $0.0472(N=17)$ \\
\hline 0.8 & 0.0347 & 0.0332 & 0.0326 & 0.0322 & 0.0322 & $0.0322(N=21)$ & $0.0322(N=21)$ \\
\hline 0.9 & 0.0191 & 0.0175 & 0.0168 & 0.0163 & 0.0164 & $0.0164(N=31)$ & $0.0164(N=31)$ \\
\hline
\end{tabular}

$n$, the number of boundary nodes; $N$, the number of collocation points on the inner cylinder. 

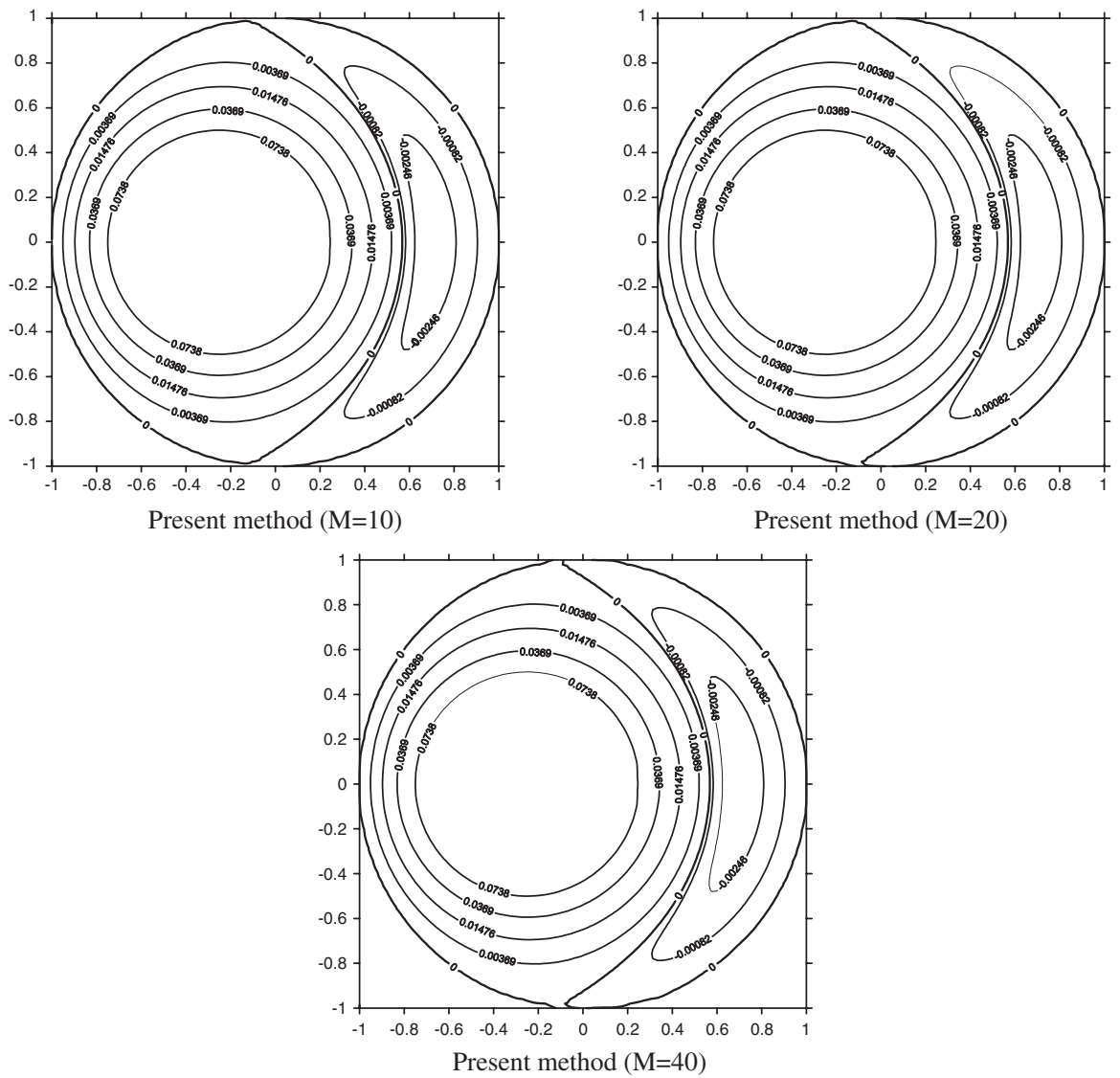

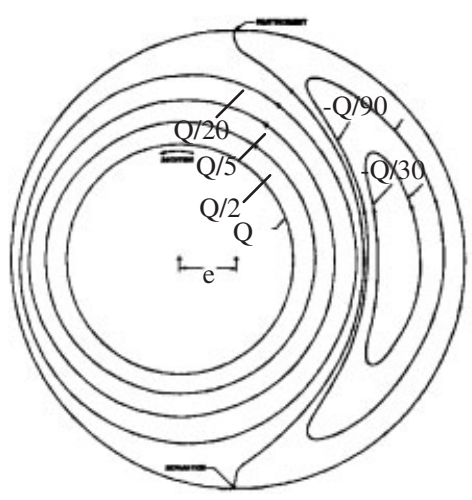

Kamal [11] Q=0.738, e=0.25

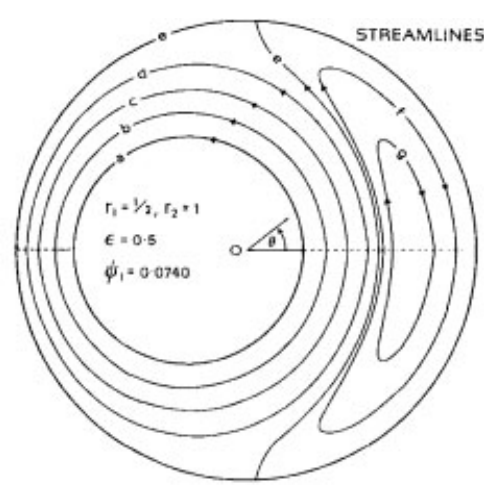

Kelmanson [14]

Figure 8. Comparison of contour plots of streamlines for $\varepsilon=0.5$. 

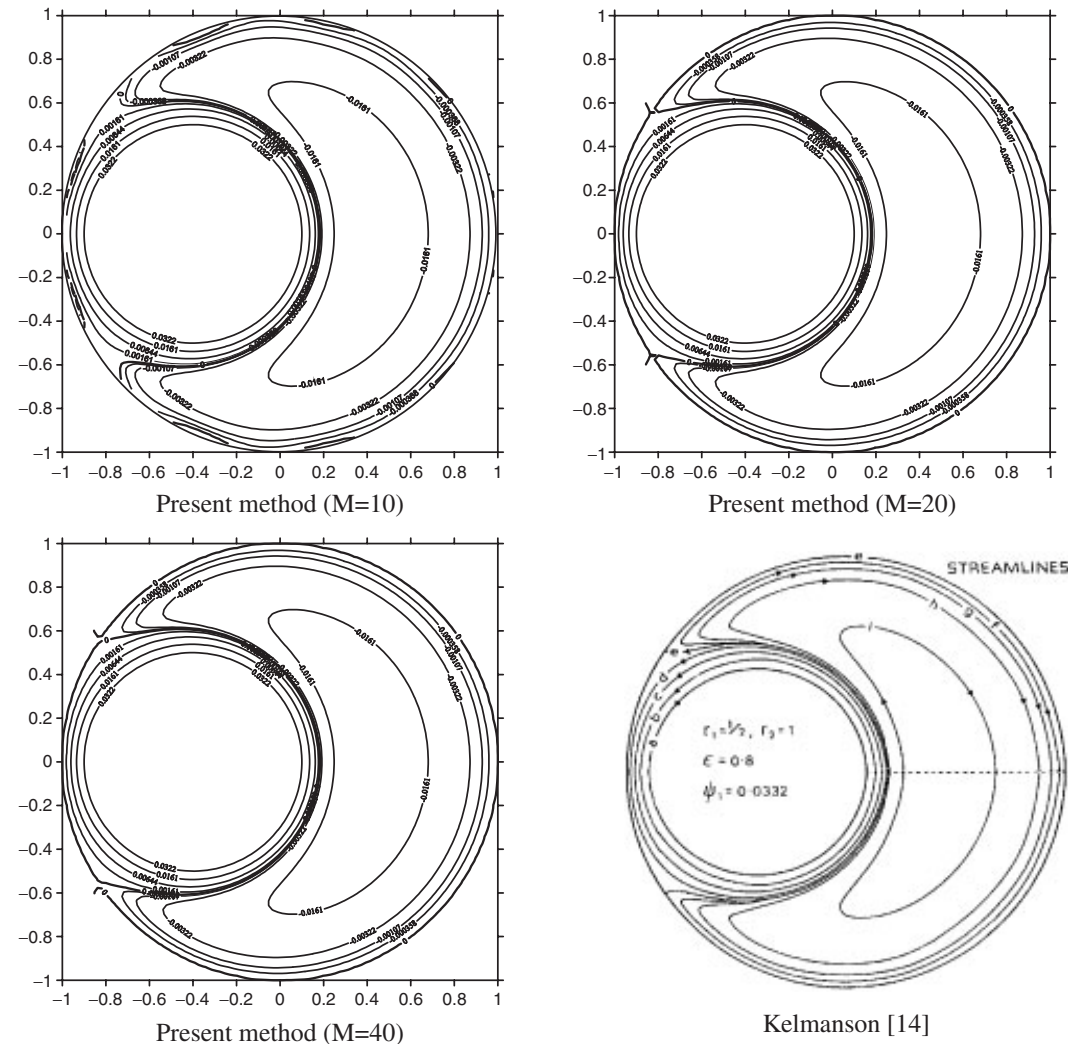

Figure 9. Comparison of streamlines contour plots for $\varepsilon=0.8$.

The flow between eccentric cylinders satisfies the biharmonic equation and the essential boundary conditions are specified as follows:

$$
\begin{aligned}
& u(s)=u_{1}, \quad \theta(s)=\frac{\partial u(s)}{\partial n}=\omega_{1} r_{1}=0.5, \quad s \text { on } B_{1} \\
& u(s)=0, \quad \theta(s)=\frac{\partial u(s)}{\partial n}=0, \quad s \text { on } B_{2}
\end{aligned}
$$

First, the direct BIEM is used. The unknown boundary densities $m(s), v(s)$ on $B_{1}$ and $m(s), v(s)$ on $B_{2}$ are expressed in terms of Fourier series. The unknown Fourier coefficients can be determined by using the null-field integral equations in conjunction with degenerate kernels and Fourier series; however, the boundary condition $u_{1}$ is an unknown constant along the inner boundary. An additional constraint is required to ensure a unique solution. From the solution procedures of the direct BIEM, $u_{1}$ with different eccentricities are calculated and the results are shown in Table I. By using the 


\section{A NEW METHOD FOR STOKES PROBLEMS WITH CIRCULAR BOUNDARIES}
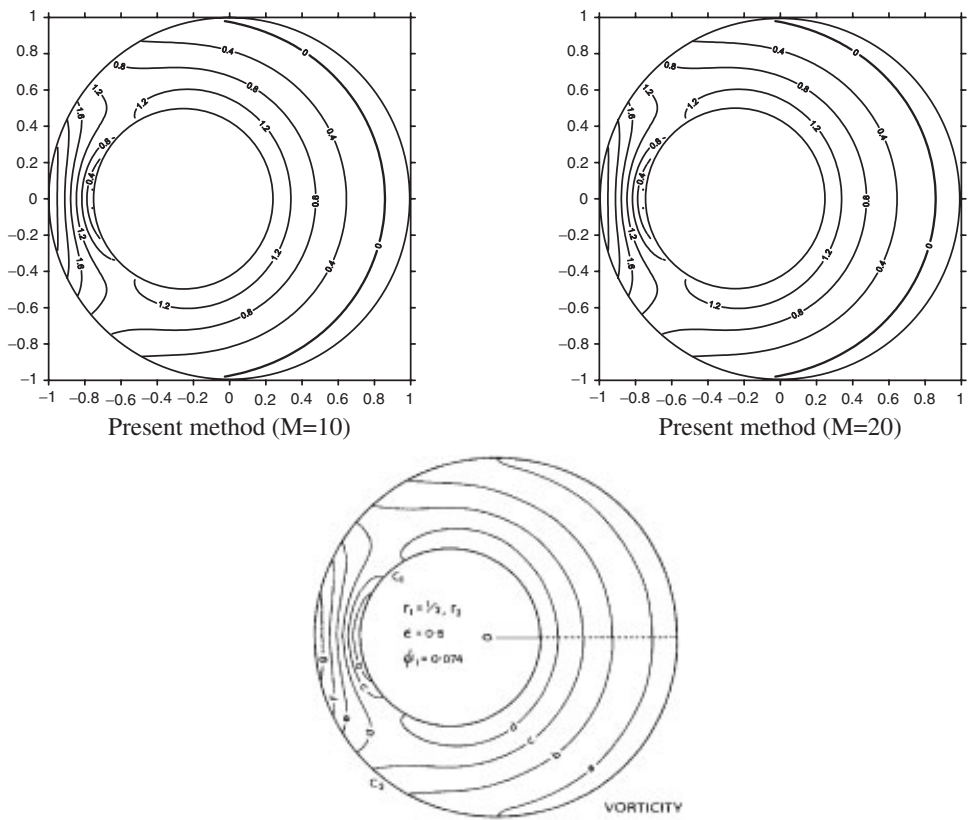

Kelmanson [14] (a) 0, (b) 0.4, (c) 0.8, (d) 1.2, (e) 1.6, (f) 2.0, (g) 2.5

Figure 10. Comparison of vorticity contour plots for $\varepsilon=0.5$.
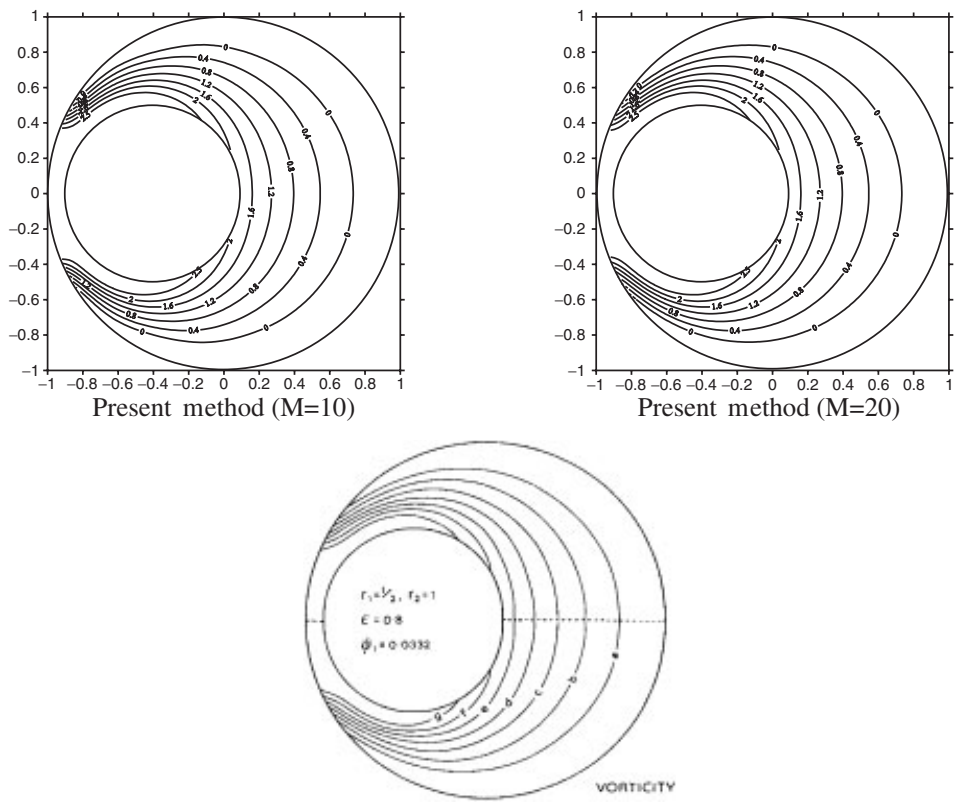

Kelmanson [14] (a) 0, (b) 0.4, (c) 0.8,(d) 1.2, (e) 1.6, (f)2.0, (g) 2.5

Figure 11. Comparison of vorticity contour plots for $\varepsilon=0.8$. 
J.-T. CHEN, C.-C. HSIAO AND S.-Y. LEU

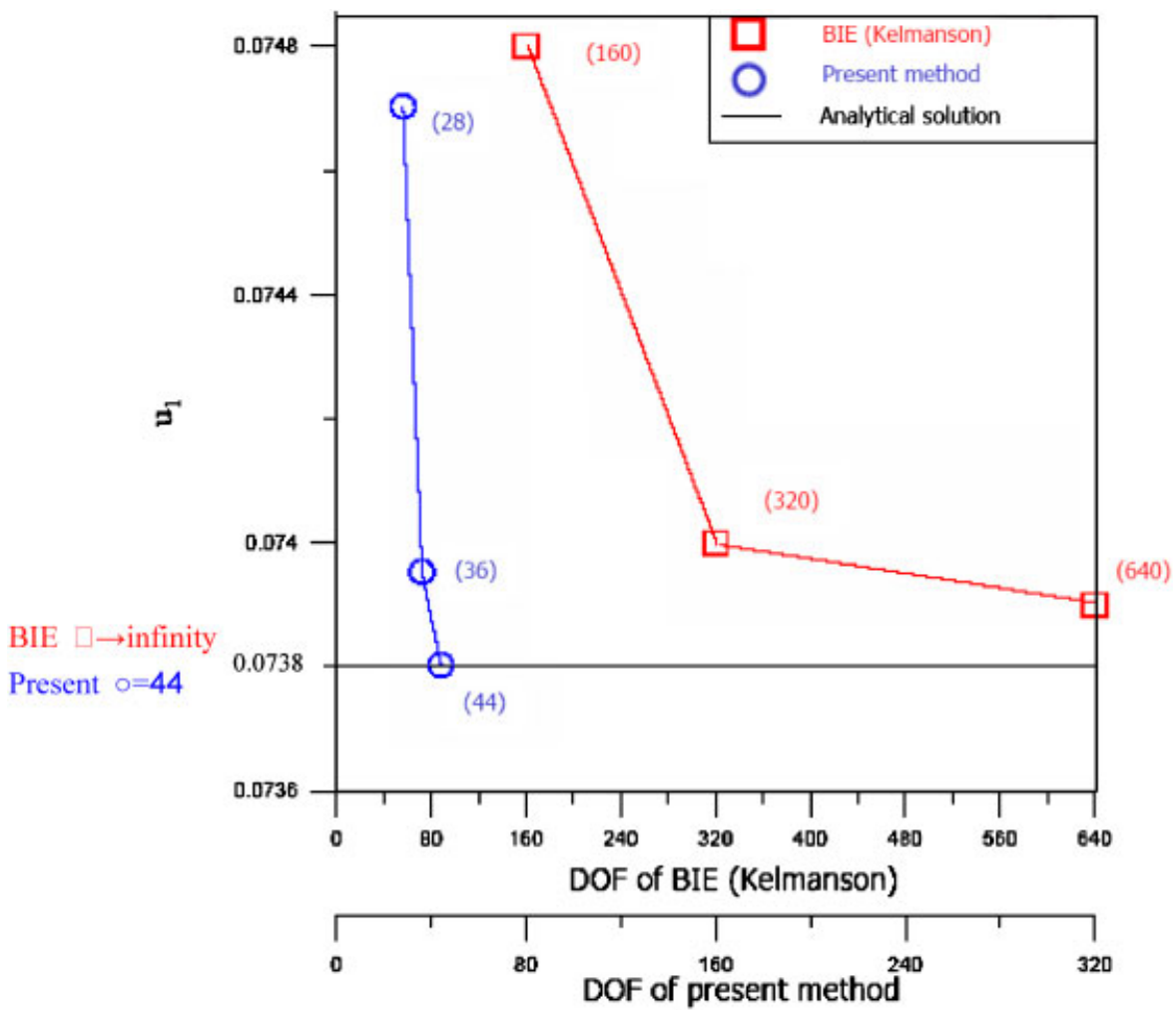

Figure 12. Comparison for $\varepsilon=0.5$ using direct formulation.

fewer degrees of freedom than BIE [14], present results are more accurate after comparing with the analytical solution as follows:

$$
u_{1}=\frac{A \omega_{1} r_{1}(\sinh \delta-\delta \cosh \delta)\left(\sinh \alpha_{2} \sinh \delta-\delta \sinh \alpha_{1}\right)}{2\left[\left(\delta+\sinh \alpha_{1} \cosh \alpha_{1}-\cosh \alpha_{2} \sinh \alpha_{2}\right)(\sinh \delta-\delta \cosh \delta)+\cosh \delta\left(\delta^{2}-\sinh \delta^{2}\right)\right]}
$$

where

$$
\begin{aligned}
& A=\frac{c}{\varepsilon}\left[\left(1-\varepsilon^{2}\right)\left[\left(\frac{r_{1}+r_{2}}{c}\right)^{2}-\varepsilon^{2}\right]\right]^{1 / 2} \\
& \alpha_{1}=-\sinh ^{-1}\left(\frac{A}{2 r_{1}}\right) \\
& \alpha_{2}=-\sinh ^{-1}\left(\frac{A}{2 r_{2}}\right) \\
& \delta=\alpha_{1}-\alpha_{2}
\end{aligned}
$$




\section{A NEW METHOD FOR STOKES PROBLEMS WITH CIRCULAR BOUNDARIES}

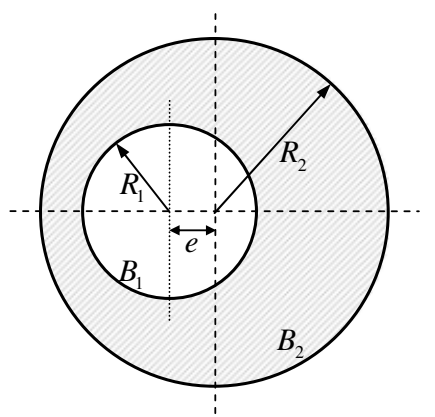

\section{Geometric data:}

$R_{1}=0.5, R_{2}=1, e=0.25$

\section{Boundary conditions:}

$u_{1}(s)=0.0738$ and $\theta_{1}(s)=0.5$ on $B_{1}$ $u_{2}(s)=0$ and $\theta_{2}(s)=0$ on $B_{2}$
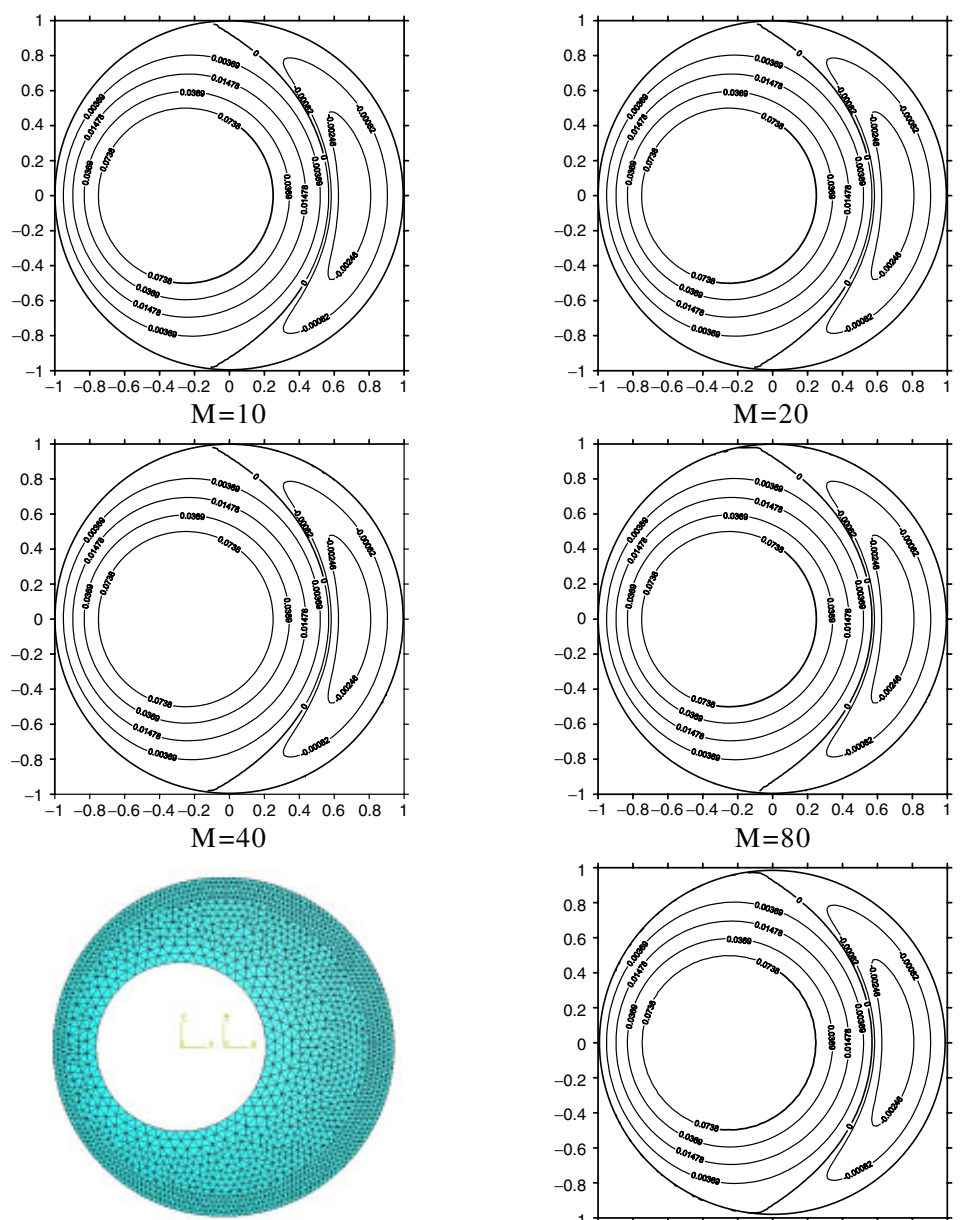

FEM mesh $($ No. of nodes $=1,734$

No. of elements $=3,218$ )

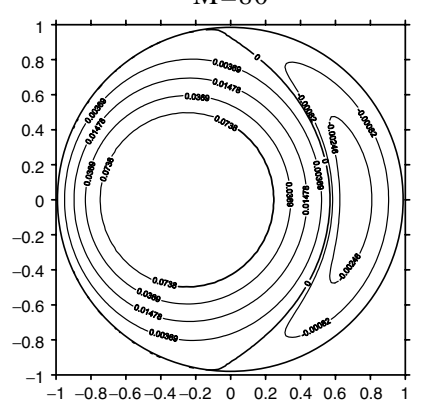

FEM result

Figure 13. The streamlines contour plot for $\varepsilon=0.5$ by using the indirect BIEM. 


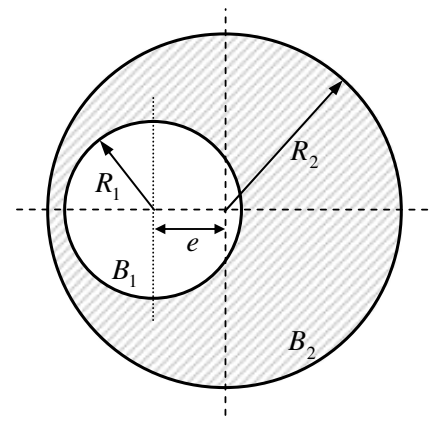

\section{Geometric data:}

$$
R_{1}=0.5, R_{2}=1, e=0.4
$$

\section{Boundary conditions:}

$u_{1}(s)=0.0322$ and $\theta_{1}(s)=0.5$ on $B_{1}$ $u_{2}(s)=0$ and $\theta_{2}(s)=0$ on $B_{2}$
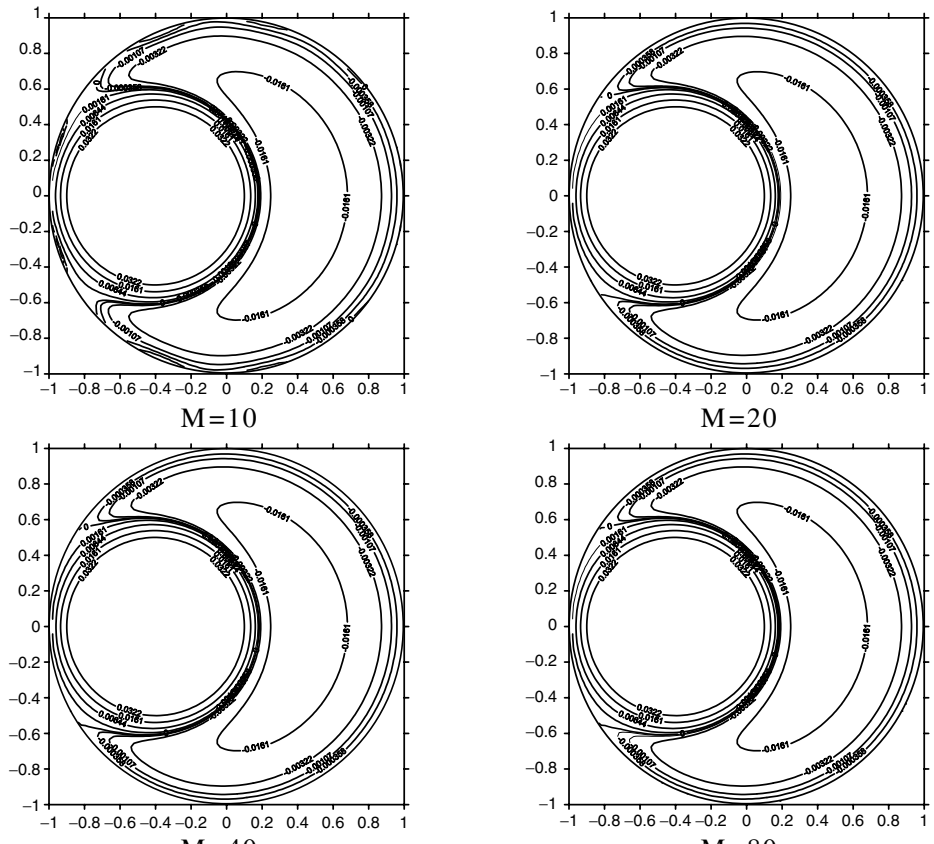

$\mathrm{M}=40$

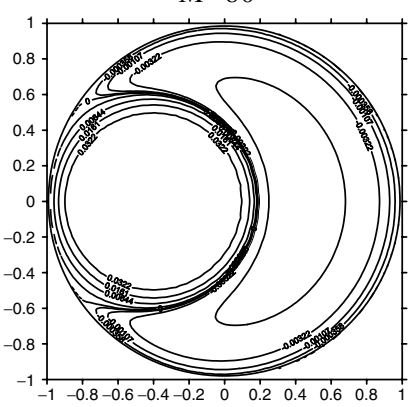

FEM result

FEM mesh (No. of nodes $=1,196$

No. of elements $=2,162$ )

Figure 14. The streamlines contour plot for $\varepsilon=0.8$ by using the indirect BIEM. 
It is noted that the number of segments $N$ in integrating on $B_{1}$ boundary of Equation (68) is the same as the number of $(2 M+1)$ collocation null-field points near the inner cylinder boundary. The contour plot of stream function and vorticity can be obtained by substituting Fourier coefficients into the BIE for the domain point of Equations (9) and (18). The streamlines and vorticity contour plots for $\varepsilon=0.5$ and 0.8 solved by employing the direct BIEM are compared with the Kelmanson's results [14] obtained by using the 160 boundary nodes and Kamal's result [11] as shown in Figures $8-11$. Figure 12 shows the rate of convergence between the present approach and BIE. It indicates that our approach shows exponential convergence rate.

According to the indirect BIEM, the unknown boundary constant $u_{1}$ for the eccentric bearing problem is also obtained as shown in Table I. Good agreement is also made after comparing the results for $\varepsilon=0.5$ and 0.8 as shown in Figures 13 and 14. Besides, the FEM by using ABAQUS software [18] is used to solve the problem and the results are also shown in Figures 13 and 14 for comparison.

\section{CONCLUDING REMARKS}

In this paper, the direct and indirect formulations in conjunction with the degenerate kernels and Fourier series expansion in adaptive observer system were proposed to solve the Stokes flow problems. ABAQUS software was also used to solve the stream function for the eccentric bearing case. The constant stream function along the inner rotating cylinder is obtained by using direct and indirect BIEMs. Only fewer numbers of collocation and segments were used to show the good agreement after comparing with the BIE results on the base of analytical solution. Although the Poisson's ratio is contained in the direct BIEM, this method can be applied to solve the Stokes problems no matter how the Poisson's ratio is specified. Although the indirect BIEM cannot provide null-field integral equation, the present method by moving the interior point to the boundary can be implemented by choosing the appropriate expansion of degenerate kernels. Numerical examples were demonstrated to see the validity of the present formulation with five gains: meshless approach, boundary-layer effect free, singularity free, exponential convergence and well-posed model.

\section{APPENDIX A: DEGENERATE KERNELS}

\section{A.1. Degenerate kernels for $U, \Theta, M, V$ in the first BIE}

$$
U(s, x)=\left\{\begin{aligned}
U^{\mathrm{I}}(s, x)= & \rho^{2}(1+\ln R)+R^{2} \ln R-\left[R \rho(1+2 \ln R)+\frac{1}{2} \frac{\rho^{3}}{R}\right] \cos (\theta-\phi) \\
& -\sum_{m=2}^{\infty}\left[\frac{1}{m(m+1)} \frac{\rho^{m+2}}{R^{m}}-\frac{1}{m(m-1)} \frac{\rho^{m}}{R^{m-2}}\right] \cos [m(\theta-\phi)], \quad R \geqslant \rho \\
U^{\mathrm{E}}(s, x)= & R^{2}(1+\ln \rho)+\rho^{2} \ln \rho-\left[\rho R(1+2 \ln \rho)+\frac{1}{2} \frac{R^{3}}{\rho}\right] \cos (\theta-\phi) \\
& -\sum_{m=2}^{\infty}\left[\frac{1}{m(m+1)} \frac{R^{m+2}}{\rho^{m}}-\frac{1}{m(m-1)} \frac{R^{m}}{\rho^{m-2}}\right] \cos [m(\theta-\phi)], \quad \rho>R
\end{aligned}\right.
$$




$$
\begin{aligned}
& \Theta(s, x)=\left\{\begin{array}{rlrl}
\Theta^{\mathrm{I}}(s, x)= & \frac{\rho^{2}}{R}+R(1+2 \ln R)-\left[\rho(3+2 \ln R)-\frac{1}{2} \frac{\rho^{3}}{R^{2}}\right] \cos (\theta-\phi) & \\
& +\sum_{m=2}^{\infty}\left[\frac{1}{m+1} \frac{\rho^{m+2}}{R^{m+1}}-\frac{m-2}{m(m-1)} \frac{\rho^{m}}{R^{m-1}}\right] \cos [m(\theta-\phi)], \quad R \geqslant \rho \\
\Theta^{\mathrm{E}}(s, x)= & 2 R(1+\ln \rho)-\left[\rho(1+2 \ln \rho)+\frac{3}{2} \frac{R^{2}}{\rho}\right] \cos (\theta-\phi) & \\
& -\sum_{m=2}^{\infty}\left[\frac{m+2}{m(m+1)} \frac{R^{m+1}}{\rho^{m}}-\frac{1}{m-1} \frac{R^{m-1}}{\rho^{m-2}}\right] \cos [m(\theta-\phi)], \quad \rho>R
\end{array}\right. \\
& \int M^{\mathrm{I}}(s, x)=(v-1) \frac{\rho^{2}}{R^{2}}+(v+3)+2(v+1) \ln R-\left[(v+1) \frac{2 \rho}{R}-(v-1) \frac{\rho^{3}}{R^{3}}\right] \cos (\theta-\phi) \\
& M(s, x)=\left\{\begin{aligned}
& +\sum_{m=2}^{\infty}\left[(v-1) \frac{\rho^{m+2}}{R^{m+2}}+\frac{m(1-v)-2(1+v)}{m} \frac{\rho^{m}}{R^{m}}\right] \cos [m(\theta-\phi)], \quad R \geqslant \rho \\
M^{\mathrm{E}}(s, x)= & 2(1+v)(1+\ln \rho)-(v+3) \frac{R}{\rho} \cos (\theta-\phi)
\end{aligned}\right. \\
& +\sum_{m=2}^{\infty}\left[\frac{m(v-1)-2(v+1)}{m} \frac{R^{m}}{\rho^{m}}+(1-v) \frac{R^{m-2}}{\rho^{m-2}}\right] \cos [m(\theta-\phi)], \quad \rho>R \\
& V(s, x)=\left\{\begin{array}{rlrl}
V^{\mathrm{I}}(s, x)= & \frac{4}{R}+\left[\frac{2 \rho}{R^{2}}(3-v)-\frac{\rho^{3}}{R^{4}}(1-v)\right] \cos (\theta-\phi) \\
& -\sum_{m=2}^{\infty}\left[m(1-v) \frac{\rho^{m+2}}{R^{m+3}}-(4+m(1-v)) \frac{\rho^{m}}{R^{m+1}}\right] \cos [m(\theta-\phi)], & R>\rho \\
V^{\mathrm{E}}(s, x)= & (-3-v) \frac{1}{\rho} \cos (\theta-\phi) \\
& +\sum_{m=2}^{\infty}\left[(m(1-v)-4) \frac{R^{m-1}}{\rho^{m}}-m(1-v) \frac{R^{m-3}}{\rho^{m-2}}\right] \cos [m(\theta-\phi)], & & \rho>R
\end{array}\right.
\end{aligned}
$$

A.2. Degenerate kernels for $U_{\theta}, \Theta_{\theta}, M_{\theta}, V_{\theta}$ in the second BIE

$$
U_{\theta}(s, x)=\left\{\begin{aligned}
U_{\theta}^{\mathrm{I}}(s, x)= & 2 \rho(1+\ln R)-\left[R(1+2 \ln R)+\frac{3}{2} \frac{\rho^{2}}{R}\right] \cos (\theta-\phi) \\
& -\sum_{m=2}^{\infty}\left[\frac{m+2}{m(m+1)} \frac{\rho^{m+1}}{R^{m}}-\frac{1}{m-1} \frac{\rho^{m-1}}{R^{m-2}}\right] \cos [m(\theta-\phi)], \quad R \geqslant \rho \\
U_{\theta}^{\mathrm{E}}(s, x)= & \frac{R^{2}}{\rho}+\rho(1+2 \ln \rho)-\left[R(3+2 \ln \rho)-\frac{1}{2} \frac{R^{3}}{\rho^{2}}\right] \cos (\theta-\phi) \\
& +\sum_{m=2}^{\infty}\left[\frac{1}{m+1} \frac{R^{m+2}}{\rho^{m+1}}-\frac{m-2}{m(m-1)} \frac{R^{m}}{\rho^{m-1}}\right] \cos [m(\theta-\phi)], \quad \rho>R
\end{aligned}\right.
$$


A NEW METHOD FOR STOKES PROBLEMS WITH CIRCULAR BOUNDARIES

$$
\begin{aligned}
& \Theta_{\theta}(s, x)=\left\{\begin{aligned}
\Theta_{\theta}^{\mathrm{I}}(s, x)= & \frac{2 \rho}{R}-\left[(3+2 \ln R)-\frac{3}{2} \frac{\rho^{2}}{R^{2}}\right] \cos (\theta-\phi) \\
& +\sum_{m=2}^{\infty}\left[\frac{m+2}{m+1} \frac{\rho^{m+1}}{R^{m+1}}-\frac{m-2}{m-1} \frac{\rho^{m-1}}{R^{m-1}}\right] \cos [m(\theta-\phi)], \quad R \geqslant \rho \\
\Theta_{\theta}^{\mathrm{E}}(s, x)= & \frac{2 R}{\rho}-\left[(3+2 \ln \rho)-\frac{3}{2} \frac{R^{2}}{\rho^{2}}\right] \cos (\theta-\phi) \\
& +\sum_{m=2}^{\infty}\left[\frac{m+2}{m+1} \frac{R^{m+1}}{\rho^{m+1}}-\frac{m-2}{m-1} \frac{R^{m-1}}{\rho^{m-1}}\right] \cos [m(\theta-\phi)], \quad \rho>R
\end{aligned}\right. \\
& M_{\theta}(s, x)=\left\{\begin{aligned}
M_{\theta}^{\mathrm{I}}(s, x)= & \frac{2 \rho}{R^{2}}(v-1)-\left[\frac{2}{R}(v+1)-3(v-1) \frac{\rho^{2}}{R^{3}}\right] \cos (\theta-\phi) \\
& +\sum_{m=2}^{\infty}\left[(m+2)(v-1) \frac{\rho^{m+1}}{R^{m+2}}+(m(1-v)-2(1+v)) \frac{\rho^{m-1}}{R^{m}}\right] \\
& \times \cos [m(\theta-\phi)], \quad R>\rho \\
M_{\theta}^{\mathrm{E}}(s, x)= & \frac{2(1+v)}{\rho}+(v+3) \frac{R}{\rho^{2}} \cos (\theta-\phi) \\
& -\sum_{m=2}^{\infty}\left[(m(v-1)-2(v+1)) \frac{R^{m}}{\rho^{m+1}}+(m-2)(1-v) \frac{R^{m-2}}{\rho^{m-1}}\right] \\
& \times \cos [m(\theta-\phi)], \quad \rho>R \quad
\end{aligned}\right. \\
& V_{\theta}(s, x)=\left\{\begin{aligned}
V_{\theta}^{\mathrm{I}}(s, x)= & {\left[\frac{2}{R^{2}}(3-v)-3(1-v) \frac{\rho^{2}}{R^{4}}\right] \cos (\theta-\phi) } \\
& -\sum_{m=2}^{\infty}\left[m(m+2)(1-v) \frac{\rho^{m+1}}{R^{m+3}}-m(4+m(1-v)) \frac{\rho^{m-1}}{R^{m+1}}\right] \\
& \times \cos [m(\theta-\phi)], \quad R>\rho \\
V_{\theta}^{\mathrm{E}}(s, x)= & (3+v) \frac{1}{\rho^{2}} \cos (\theta-\phi) \\
& -\sum_{m=2}^{\infty}\left[m(m(1-v)-4) \frac{R^{m-1}}{\rho^{m+1}}-m(m-2)(1-v) \frac{R^{m-3}}{\rho^{m-1}}\right] \\
& \times \cos [m(\theta-\phi)], \quad \rho>R
\end{aligned}\right.
\end{aligned}
$$

where $U_{\theta}, \Theta_{\theta}, M_{\theta}, V_{\theta}$ are equal to $\partial U(s, x) / \partial n_{x}, \partial \Theta(s, x) / \partial n_{x}, \partial M(s, x) / \partial n_{x}$ and $\partial V(s, x) / \partial n_{x}$, respectively. 


\section{J.-T. CHEN, C.-C. HSIAO AND S.-Y. LEU}

\section{A.3. Tangential derivative with respect to the field point}

$$
\begin{aligned}
& U_{, t}(s, x)=\left\{\begin{array}{rlrl}
U_{, t}^{\mathrm{I}}(s, x)= & -\left[R(1+2 \ln R)+\frac{1}{2} \frac{\rho^{2}}{R}\right] \sin (\theta-\phi) & \\
& -\sum_{m=2}^{\infty}\left[\frac{1}{m+1} \frac{\rho^{m+1}}{R^{m}}-\frac{1}{m-1} \frac{\rho^{m-1}}{R^{m-2}}\right] \sin [m(\theta-\phi)], \quad R>\rho \\
U_{, t}^{\mathrm{E}}(s, x)= & -\left[R(1+2 \ln \rho)+\frac{1}{2} \frac{R^{3}}{\rho^{2}}\right] \sin (\theta-\phi) & \\
& -\sum_{m=2}^{\infty}\left[\frac{1}{m+1} \frac{R^{m+2}}{\rho^{m+1}}-\frac{1}{m-1} \frac{R^{m}}{\rho^{m-1}}\right] \sin [m(\theta-\phi)], \quad \rho>R
\end{array}\right. \\
& \Theta_{, t}(s, x)=\left\{\begin{aligned}
\Theta_{, t}^{\mathrm{I}}(s, x)= & -\left(3+2 \ln R-\frac{1}{2} \frac{\rho^{2}}{R^{2}}\right) \sin (\theta-\phi) \\
& +\sum_{m=2}^{\infty}\left[\frac{m}{m+1} \frac{\rho^{m+1}}{R^{m+1}}-\frac{m-2}{m-1} \frac{\rho^{m-1}}{R^{m-1}}\right] \sin [m(\theta-\phi)], \quad R>\rho \\
\Theta_{, t}^{\mathrm{E}}(s, x)= & -\left(1+2 \ln \rho+\frac{3}{2} \frac{R^{2}}{\rho^{2}}\right) \sin (\theta-\phi) \\
& -\sum_{m=2}^{\infty}\left[\frac{m+2}{m+1} \frac{R^{m+1}}{\rho^{m+1}}-\frac{m}{m-1} \frac{R^{m-1}}{\rho^{m-1}}\right] \sin [m(\theta-\phi)], \quad \rho>R
\end{aligned}\right. \\
& M_{, t}(s, x)=\left\{\begin{aligned}
M_{, t}^{\mathrm{I}}(s, x)= & -\left[\frac{2(v+1)}{R}-(v-1) \frac{\rho^{2}}{R^{3}}\right] \sin (\theta-\phi) \\
& +\sum_{m=2}^{\infty}\left[m(v-1) \frac{\rho^{m+1}}{R^{m+2}}+(m(1-v)-2(1+v)) \frac{\rho^{m-1}}{R^{m}}\right] \sin [m(\theta-\phi)], \quad R>\rho \\
M_{, t}^{E}(s, x)= & -(v+3) \frac{R}{\rho^{2}} \sin (\theta-\phi) \\
& +\sum_{m=2}^{\infty}\left[(m(v-1)-2(v+1)) \frac{R^{m}}{\rho^{m+1}}+m(1-v) \frac{R^{m-2}}{\rho^{m-1}}\right] \sin [m(\theta-\phi)], \quad \rho>R
\end{aligned}\right. \\
& V_{, t}(s, x)=\left\{\begin{aligned}
V_{, t}^{\mathrm{I}}(s, x)= & {\left[\frac{2(3-v)}{R^{2}}-\frac{\rho^{2}}{R^{4}}(1-v)\right] \sin (\theta-\phi) } \\
& -\sum_{m=2}^{\infty}\left[m^{2}(1-v) \frac{\rho^{m+1}}{R^{m+3}}-m(4+m(1-v)) \frac{\rho^{m-1}}{R^{m+1}}\right] \sin [m(\theta-\phi)], \quad R>\rho \\
V_{, t}^{E}(s, x)= & (-3-v) \frac{1}{\rho^{2}} \sin (\theta-\phi)+\sum_{m=2}^{\infty}\left[m(m(1-v)-4) \frac{R^{m-1}}{\rho^{m+1}}\right. \\
& \left.-m^{2}(1-v) \frac{R^{m-3}}{\rho^{m-1}}\right] \sin [m(\theta-\phi)], \quad \rho>R
\end{aligned}\right.
\end{aligned}
$$




\section{A NEW METHOD FOR STOKES PROBLEMS WITH CIRCULAR BOUNDARIES}

A.4. Laplacian of the degenerate kernels with respect to $U, \Theta, M, V$

$$
\begin{aligned}
& U_{\nabla^{2}}(s, x)= \begin{cases}U_{\nabla^{2}}^{\mathrm{I}}(s, x)=4(1+\ln R)-4 \frac{\rho}{R} \cos (\theta-\phi)-\sum_{m=2}^{\infty} \frac{4}{m} \frac{\rho^{m}}{R^{m}} \cos [m(\theta-\phi)], & R>\rho \\
U_{\nabla^{2}}^{\mathrm{E}}(s, x)=4(1+\ln \rho)-4 \frac{R}{\rho} \cos (\theta-\phi)-\sum_{m=2}^{\infty} \frac{4}{m} \frac{R^{m}}{\rho^{m}} \cos [m(\theta-\phi)], & \rho>R\end{cases} \\
& \Theta_{\nabla^{2}}(s, x)= \begin{cases}\Theta_{\nabla^{2}}^{\mathrm{I}}(s, x)=\frac{4}{R}+4 \frac{\rho}{R^{2}} \cos (\theta-\phi)+\sum_{m=2}^{\infty} 4 \frac{\rho^{m}}{R^{m+1}} \cos [m(\theta-\phi)], & R>\rho \\
\Theta_{\nabla^{2}}^{\mathrm{E}}(s, x)=-\frac{4}{\rho} \cos (\theta-\phi)-\sum_{m=2}^{\infty} 4 \frac{R^{m-1}}{\rho^{m}} \cos [m(\theta-\phi)], & \rho>R\end{cases} \\
& M_{\nabla^{2}}(s, x)=\left\{\begin{aligned}
M_{\nabla^{2}}^{\mathrm{I}}(s, x)= & \frac{4}{R^{2}}(v-1)+8(v-1) \frac{\rho}{R^{3}} \cos (\theta-\phi) & & \\
& +\sum_{m=2}^{\infty} 4(m+1)(v-1) \frac{\rho^{m}}{R^{m+2}} \cos [m(\theta-\phi)], & & R>\rho \\
M_{\nabla^{2}}^{\mathrm{E}}(s, x)= & \sum_{m=2}^{\infty} 4(m-1)(v-1) \frac{R^{m-2}}{\rho^{m}} \cos [m(\theta-\phi)], & & \rho>R
\end{aligned}\right. \\
& V_{\nabla^{2}}(s, x)=\left\{\begin{array}{rlrl}
V_{\nabla^{2}}^{\mathrm{I}}(s, x)= & 8(v-1) \frac{\rho}{R^{4}} \cos (\theta-\phi) & \\
& +\sum_{m=2}^{\infty} 4 m(m+1)(v-1) \frac{\rho^{m}}{R^{m+3}} \cos [m(\theta-\phi)], & & R>\rho \\
V_{\nabla^{2}}^{\mathrm{E}}(s, x)= & -\sum_{m=2}^{\infty} 4 m(m-1)(v-1) \frac{R^{m-3}}{\rho^{m}} \cos [m(\theta-\phi)], & & \rho>R
\end{array}\right.
\end{aligned}
$$

\section{A.5. Normal derivative of Laplacian of the degenerate kernels}

$$
\begin{aligned}
& U_{\nabla^{2}, n}(s, x)= \begin{cases}U_{\nabla^{2}, n}^{\mathrm{I}}(s, x)=-\frac{4}{R} \cos (\theta-\phi)-\sum_{m=2}^{\infty} 4 \frac{\rho^{m-1}}{R^{m}} \cos [m(\theta-\phi)], & R>\rho \\
U_{\nabla^{2}, n}^{\mathrm{E}}(s, x)=\frac{4}{\rho}+4 \frac{R}{\rho^{2}} \cos (\theta-\phi)+\sum_{m=2}^{\infty} 4 \frac{R^{m}}{\rho^{m+1}} \cos [m(\theta-\phi)], & \rho>R\end{cases} \\
& \Theta_{\nabla^{2}, n}(s, x)= \begin{cases}\Theta_{\nabla^{2}, n}^{\mathrm{I}}(s, x)=\frac{4}{R^{2}} \cos (\theta-\phi)+\sum_{m=2}^{\infty} 4 m \frac{\rho^{m-1}}{R^{m+1}} \cos [m(\theta-\phi)], & R>\rho \\
\Theta_{\nabla^{2}, n}^{\mathrm{E}}(s, x)=\frac{4}{\rho^{2}} \cos (\theta-\phi)+\sum_{m=2}^{\infty} 4 m \frac{R^{m-1}}{\rho^{m+1}} \cos [m(\theta-\phi)], & \rho>R\end{cases}
\end{aligned}
$$




$$
\begin{aligned}
M_{\nabla^{2}, n}(s, x)=\left\{\begin{array}{rlr}
M_{\nabla^{2}, n}^{\mathrm{I}}(s, x)= & \frac{8(v-1)}{R^{3} \cos (\theta-\phi)} \\
& +\sum_{m=2}^{\infty} 4 m(m+1)(v-1) \frac{\rho^{m-1}}{R^{m+2}} \cos [m(\theta-\phi)], & R>\rho \\
M_{\nabla^{2}, n}^{\mathrm{E}}(s, x)=-\sum_{m=2}^{\infty} 4 m(m-1)(v-1) \frac{R^{m-2}}{\rho^{m+1}} \cos [m(\theta-\phi)], & & \rho>R
\end{array}\right. \\
V_{\nabla^{2}, n}(s, x)=\left\{\begin{array}{rlr}
V_{\nabla^{2}, n}^{\mathrm{I}}(s, x)= & \frac{8(v-1)}{R^{4} \cos (\theta-\phi)} \\
& +\sum_{m=2}^{\infty} 4 m^{2}(m+1)(v-1) \frac{\rho^{m-1}}{R^{m+3}} \cos [m(\theta-\phi)], & R>\rho \\
V_{\nabla^{2}, n}^{\mathrm{E}}(s, x)=\sum_{m=2}^{\infty} 4 m^{2}(m-1)(v-1) \frac{R^{m-3}}{\rho^{m+1}} \cos [m(\theta-\phi)], & & \rho>R
\end{array}\right.
\end{aligned}
$$

A.6. Tangential derivative of Laplacian of the degenerate kernels

$$
\begin{aligned}
& U_{\nabla^{2}, t}(s, x)= \begin{cases}U_{\nabla^{2}, t}^{\mathrm{I}}(s, x)=-\frac{4}{R} \sin (\theta-\phi)-\sum_{m=2}^{\infty} 4 \frac{\rho^{m-1}}{R^{m}} \sin [m(\theta-\phi)], & R>\rho \\
U_{\nabla^{2}, t}^{\mathrm{E}}(s, x)=-4 \frac{R}{\rho^{2}} \sin (\theta-\phi)-\sum_{m=2}^{\infty} 4 \frac{R^{m}}{\rho^{m+1}} \sin [m(\theta-\phi)], & \rho>R\end{cases} \\
& \Theta_{\nabla^{2}, t}(s, x)= \begin{cases}\Theta_{\nabla^{2}, t}^{\mathrm{I}}(s, x)=\frac{4}{R^{2}} \sin (\theta-\phi)+\sum_{m=2}^{\infty} 4 m \frac{\rho^{m-1}}{R^{m+1}} \sin [m(\theta-\phi)], & R>\rho \\
\Theta_{\nabla^{2}, t}^{\mathrm{E}}(s, x)=-\frac{4}{\rho^{2}} \sin (\theta-\phi)-\sum_{m=2}^{\infty} 4 m \frac{R^{m-1}}{\rho^{m+1}} \cos [m(\theta-\phi)], & \rho>R\end{cases} \\
& M_{\nabla^{2}, t}(s, x)=\left\{\begin{aligned}
& M_{\nabla^{2}, t}^{\mathrm{I}}(s, x)=\frac{8(v-1)}{R^{3}} \sin (\theta-\phi) R>\rho \\
&+\sum_{m=2}^{\infty} 4 m(m+1)(v-1) \frac{\rho^{m-1}}{R^{m+2}} \sin [m(\theta-\phi)], R>R \\
& M_{\nabla^{2}, t}^{\mathrm{E}}(s, x)=\sum_{m=2}^{\infty} 4 m(m-1)(v-1) \frac{R^{m-2}}{\rho^{m+1}} \sin [m(\theta-\phi)], \rho>R \\
& V_{\nabla^{2}, t}(s, x)=\left\{\begin{aligned}
\sum_{m=2}^{\infty} 4 m^{2}(m+1)(v-1) \frac{\rho^{m-1}}{R^{m+3}} \sin [m(\theta-\phi)], & R>\rho \\
V_{\nabla^{2}, t}^{\mathrm{E}}(s, x)=-\sum_{m=2}^{\infty} 4 m^{2}(m-1)(v-1) \frac{R^{m-3}}{\rho^{m+1}} \sin [m(\theta-\phi)], & \rho>R
\end{aligned}\right.
\end{aligned}\right.
\end{aligned}
$$




\section{A NEW METHOD FOR STOKES PROBLEMS WITH CIRCULAR BOUNDARIES}

\section{REFERENCES}

1. Sladek J, Sladek V. Three-dimensional crack analysis for anisotropic body. Applied Mathematical Modelling 1982; 6:374-380.

2. Chen JT, Wu CS, Chen KH. A study of free terms for plate problems in the dual boundary integral equations. Engineering Analysis with Boundary Elements 2005; 29:435-446.

3. Chen JT, Wu CS, Chen KH, Lee YT. Degenerate scale for plate analysis using the boundary integral equation method and boundary element method. Computational Mechanics 2006; 38:33-49.

4. Bird MD, Steele CR. Separated solution procedure for bending of circular plates with circular holes. Applied Mechanics Reviews 1991; 44:27-35.

5. Chen JT, Shen WC, Wu AC. Null-field integral equation for stress field around circular holes under antiplane shear. Engineering Analysis with Boundary Elements 2006; 30(3):205-217.

6. Crouch SL, Mogilevskaya SG. On the use of Somigliana's formula and Fourier series for elasticity problems with circular boundaries. International Journal for Numerical Methods in Engineering 2003; 58:537-578.

7. Mogilevskaya SG, Crouch SL. A Galerkin boundary integral method for multiple circular elastic inclusions. International Journal for Numerical Methods in Engineering 2001; 52:1069-1106.

8. Mills RD. Computing internal viscous flow problems for the circle by integral methods. Journal of Fluid Mechanics 1977; 73:609-624.

9. Muldowney GP, Higdon JJL. A spectral boundary element approach to three-dimensional Stokes flow. Journal of Fluid Mechanics 1995; 298:167-192.

10. Youngren GK, Acrivos A. Stokes flow past a particle of arbitrary shape: a numerical method of solution. Journal of Fluid Mechanics 1975; 69:377-403.

11. Kamal MM. Separation in the flow between eccentric rotating cylinders. Journal of Basic Engineering 1966; D88:717-724.

12. DiPrima RC, Stuart JT. Flow between eccentric rotating cylinders. Journal of Lubrication Technology 1972; 94:266-274.

13. Ingham DB, Kelmanson MA. Boundary Integral Equation Analyses of Singular, Potential, and Biharmonic Problems. Lecture Notes in Engineering, vol. 7. Springer: Berlin, Heidelberg, 1984.

14. Kelmanson MA. A boundary integral equation method for the study of slow flow in bearings with arbitrary geometries. Journal of Tribology 1984; 106:260-264.

15. Wannier GH. A contribution to the hydrodynamics of lubrication. Quarterly of Applied Mathematics 1950; 8:1-32.

16. Pozrikidis C. Boundary Integral and Singularity Methods for Linearized Viscous Flow. Cambridge University Press: Cambridge, 1992.

17. Hsiao CC. A semi-analytical approach for Stokes flow and plate problems with circular boundaries. Master Thesis, Department of Harbor and River Engineering, National Taiwan Ocean University, Keelung, Taiwan, 2005.

18. ABAQUS/CAE 6.5, Hibbitt, Karlsson and Sorensen, Inc., RI, 2004. 